\title{
Dynamic Optimization and Learning for Renewal Systems
}

\author{
Michael J. Neely
}

\begin{abstract}
We consider the problem of optimizing time averages in systems with independent and identically distributed behavior over renewal frames. This includes scheduling and task processing to maximize utility in stochastic networks with variable length scheduling modes. Every frame, a new policy is implemented that affects the frame size and that creates a vector of attributes. An algorithm is developed for choosing policies on each frame in order to maximize a concave function of the time average attribute vector, subject to additional time average constraints. The algorithm is based on Lyapunov optimization concepts and involves minimizing a "drift-plus-penalty" ratio over each frame. The algorithm can learn efficient behavior without a-priori statistical knowledge by sampling from the past. Our framework is applicable to a large class of problems, including Markov decision problems.
\end{abstract}

\section{INTRODUCTION}

Consider a stochastic system that regularly experiences times when the system state is refreshed, called renewal times. The goal is to develop a control algorithm that maximizes the time average of a reward process associated with the system, subject to time average constraints on a collection of penalty processes. The renewal-reward theorem is a simple and elegant technique for computing time averages in such systems (see, for example, [2][3]). However, the renewal-reward theorem requires random events to be independent and identically distributed (i.i.d.) over each renewal frame. While this i.i.d. assumption may hold if a single control law is implemented repeatedly, it is often difficult to choose in advance a single control law that optimizes the system subject to the desired constraints. This paper investigates the situation where the control policies used may differ from frame to frame, and are designed to dynamically solve the problem of interest.

This renewal problem arises in many different applications. One application of interest is a task processing network. For example, consider a network of wireless devices that repeatedly collaborate to accomplish tasks (such as reporting sensor data to a destination, or performing distributed computation on data). Tasks are performed one after the other, and for each task we must decide what modes of operation and communication to use, possibly allowing some nodes of the network to remain idle to save power. It is then important to make decisions that maximize the time average utility associated with task processing, subject to time average power constraints

Michael J. Neely is with the Electrical Engineering department at the University of Southern California, Los Angeles, CA.

This paper was presented in part at the Asilomar Conference on Signals, Systems, and Computers, Pacific Grove, CA, 2010 [1]. This material is supported in part by one or more of the following: the DARPA IT-MANET program grant W911NF-07-0028, the NSF Career grant CCF-0747525, and continuing through participation in the Network Science Collaborative Technology Alliance sponsored by the U.S. Army Research Laboratory. at each node. Alternatively, one may want to minimize time average power, subject to constraints on utility and on the "left-over" communication rates available for data that is not associated with the task processing.

This paper develops a general framework for solving such problems. To do so, we extend the theory of Lyapunov optimization from [4]. Specifically, work in [4] considers discrete time queueing networks and develops a simple drift-pluspenalty rule for making optimal decisions. These decisions are made in a greedy manner every slot based only on the observed traffic and channel conditions for that slot, without requiring a-priori knowledge of the underlying probability distribution. However, the work in [4] assumes all slots have fixed length, the random network condition is observed at the beginning of each slot and does not change over the slot, and this condition is not influenced by control actions. The general renewal problem treated in the current paper is more complex because each frame may have a different length and may contain a sequence of random events. The frame length and the random event sequence may depend on the control decisions made over the course of the frame. Rather than making a single decision every slot, every frame we must specify a policy, being a contingency plan for making decisions over the course of the frame in reaction to the resulting system events.

This paper solves the general problem with a conceptually simple technique that chooses a policy to minimize a driftplus-penalty ratio every frame. We first develop algorithms for minimizing the time average of a penalty process subject to a collection of time average constraints. We then consider maximization of a concave function of a vector of time average attributes subject to similar constraints. This utility maximization problem is challenging because of the variable frame length. We overcome this challenge with a novel transformation together with a variation of Jensen's inequality.

While this paper focuses on task processing applications, we note that our renewal framework can also handle Markov decision problems. Specifically, suppose the system operates according to either a continuous or discrete time Markov chain with control-dependent transition probabilities. If the chain has a recurrent state, then renewals can be defined as re-visitations to this state, and the same drift-plus-penalty ratio technique can be applied. However, the drift-plus-penalty ratio may be difficult to optimize for Markov decision problems with high dimension (see also [5]).

Prior work on learning algorithms for Markov decision problems is in [6], and related work in [7][8][9][10] considers learning for optimization of energy and delay in queueing systems. The works [6]-[10] use stochastic approximation theory and two-timescale convergence analysis. The Lagrange multiplier updates in [6]-[10] are analogous to the virtual 


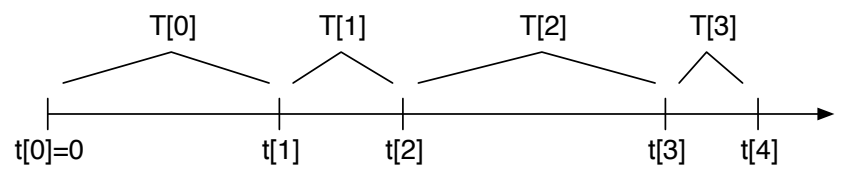

Fig. 1. A timeline illustrating renewal frames for the system.

queue updates we use in this paper. However, the Lyapunov optimization framework we use is different and does not require a two-timescale approach. It also provides more explicit bounds on convergence times and deviations from optimality, and allows a broader class of problems such as task processing problems.

The Lyapunov optimization technique that we use in this paper is based on our previous work in [4][11][12][13] that develops the drift-plus-penalty method for stochastic network optimization, including opportunistic scheduling for throughpututility maximization [4][11][13] and average power minimization [12] (see also [14]). Alternative "fluid-based" stochastic optimization techniques for queueing networks are developed in [15][16][17][18], and dual and primal-dual algorithms for systems without queues, based on tracking a corresponding static optimization problem, are in [19][20][21]. Our current paper considers the more complex renewal problem, and leverages ideas in [5][22], where [5] considers a frame-based Lyapunov framework for Markov decision problems involving network delay, and [22] develops a ratio rule for utility optimization in wireless systems with variable length frames and time-correlated channels.

Recent work in [23] considers a task processing system where multiple wireless "reporting nodes" select data formats (e.g., "voice" or "video") in which to deliver sensed information. The work [23] also uses a renewal structure. However, it assumes a single random event occurs at the beginning of each renewal frame, and the event and frame size are not influenced by control actions. More general problems can be treated using the theory developed in the current paper.

\section{Renewal System Model}

Consider a system that operates over renewal frames. Specifically, consider the timeline of non-negative real times $t \geq 0$, and suppose this timeline is segmented into successive frames of duration $\{T[0], T[1], T[2], \ldots\}$, as shown in Fig. 1 Define $t[0]=0$, and for each positive integer $r$ define $t[r]$ as the rth renewal time:

$$
t[r] \triangleq \sum_{i=0}^{r-1} T[i]
$$

The interval of all times $t$ such that $t[r] \leq t<t[r+1]$ is defined as the rth renewal frame, defined for each $r \in$ $\{0,1,2, \ldots\}$.

At the beginning of each renewal frame $r$, the controller selects a policy $\pi[r]$ from an abstract policy space $\mathcal{P}$, and implements the policy over the duration of the frame. There may be random events that arise over the renewal frame (with distributions that are possibly dependent on the policy), and the policy specifies a contingency plan for react- ing to these events. The policy incurs a vector of penalties $\boldsymbol{y}[r]=\left(y_{0}[r], y_{1}[r], \ldots, y_{L}[r]\right)$ and attributes $\boldsymbol{x}[r]=$ $\left(x_{1}[r], \ldots, x_{M}[r]\right)$ for some integers $L \geq 0, M \geq 0$ (where $L=0$ corresponds to problems without $\boldsymbol{y}[r]$ penalties, and $M=0$ corresponds to problems without $\boldsymbol{x}[r]$ attributes). The policy may also affect the renewal frame duration $T[r]$. Formally, the values $T[r], y_{l}[r], x_{m}[r]$ are determined by random functions $\hat{T}(\cdot), \hat{y}_{l}(\cdot), \hat{x}_{m}(\cdot)$ of the policy $\pi[r]$ :

$$
\begin{aligned}
T[r] & \triangleq \hat{T}(\pi[r]) \\
y_{l}[r] & \triangleq \hat{y}_{l}(\pi[r]) \forall l \in\{0,1, \ldots, L\} \\
x_{m}[r] & \triangleq \hat{x}_{m}(\pi[r]) \forall m \in\{1, \ldots, M\}
\end{aligned}
$$

We assume the values of $\left[\hat{T}(\pi[r]),\left(\hat{y}_{l}(\pi[r])\right),\left(\hat{x}_{m}(\pi[r])\right)\right]$ for frame $r$ are conditionally independent of events in previous frames given the particular policy $\pi=\pi[r]$, and are identically distributed over all frames that use the same policy $\pi$.

Consider now a particular control algorithm that chooses policies $\pi[r] \in \mathcal{P}$ every frame $r$ according to some well defined (possibly probabilistic) rule, and define the following frame-average expectations, defined for integers $R>0$ :

$$
\bar{T}[R] \triangleq \frac{1}{R} \sum_{r=0}^{R-1} \mathbb{E}\{T[r]\} \quad, \bar{y}_{l}[R] \triangleq \frac{1}{R} \sum_{r=0}^{R-1} \mathbb{E}\left\{y_{l}[r]\right\}
$$

where we recall that $T[r], y_{l}[r], x_{m}[r]$ depend on the policy $\pi[r]$ by (1)-(3). Define $\bar{x}_{m}[R]$ similarly, and define the infinite horizon frame-average expectations $\bar{T}, \bar{y}_{l}, \bar{x}_{m}$ by:

$$
\left(\bar{T}, \bar{y}_{l}, \bar{x}_{m}\right)=\lim _{R \rightarrow \infty}\left(\bar{T}[R], \bar{y}_{l}[R], \bar{x}_{m}[R]\right)
$$

where we temporarily assume the limits are well defined.

\section{A. Optimization Objective}

The first type of problem we consider uses only penalties $\boldsymbol{y}[r]$ : We must choose a policy $\pi[r] \in \mathcal{P}$ every frame $r$ to minimize the ratio $\bar{y}_{0} / \bar{T}$ subject to constraints on $\bar{y}_{l} / \bar{T}$ :

$$
\begin{array}{cc}
\text { Minimize: } & \bar{y}_{0} / \bar{T} \\
\text { Subject to: } & \bar{y}_{l} / \bar{T} \leq c_{l} \forall l \in\{1, \ldots, L\} \\
& \pi[r] \in \mathcal{P} \forall r \in\{0,1,2, \ldots\}
\end{array}
$$

where $c_{l}$ for $l \in\{1, \ldots, L\}$ are a given collection of realvalued (possibly negative) constants.

The motivation for looking at the ratio $\bar{y}_{l} / \bar{T}$ is that it defines the time average penalty associated with the $y_{l}[r]$ process. To see this, suppose the following limits converge to constants $y_{l}^{a v}$ and $T^{a v}$ with probability 1 :

$\lim _{R \rightarrow \infty} \frac{1}{R} \sum_{r=0}^{R-1} y_{l}[r]=y_{l}^{a v}, \lim _{R \rightarrow \infty} \frac{1}{R} \sum_{r=0}^{R-1} T[r]=T^{a v}$ (w.p.1)

Under very mild conditions, the existence of the limits $y_{l}^{a v}$ and $T^{a v}$ implies the frame-average expectations also have well defined limits, with $\bar{y}_{l}=y_{l}^{a v}$ and $\bar{T}=T^{a v}$. This holds, for example, whenever $y_{l}[r]$ and $T[r]$ are deterministically bounded by finite constants, or when more general conditions hold that allow the Lebesgue dominated convergence theorem to be applied [24]. Then the time average penalty per unit 
time associated with $y_{l}[r]$ (sampled only at renewal times for simplicity) satisfies with probability 1 :

$$
\lim _{R \rightarrow \infty} \frac{\sum_{r=0}^{R-1} y_{l}[r]}{\sum_{r=0}^{R-1} T[r]}=\lim _{R \rightarrow \infty} \frac{\frac{1}{R} \sum_{r=0}^{R-1} y_{l}[r]}{\frac{1}{R} \sum_{r=0}^{R-1} T[r]}=\frac{\bar{y}_{l}}{\bar{T}}
$$

Therefore, the value $\bar{y}_{l} / \bar{T}$ indeed represents the limiting penalty per unit time associated with the process $y_{l}[r]$.

The problem (5)-(7) seeks only to minimize a time average subject to time average constraints. The second problem we consider, more general than the first, seeks to maximize a concave and entrywise non-decreasing function $\phi(\gamma)$ of the time average attribute vector ratio $\overline{\boldsymbol{x}} / \bar{T}$, where $\overline{\boldsymbol{x}}=\left(\bar{x}_{1}, \ldots, \bar{x}_{M}\right)$ :

$$
\begin{array}{cc}
\text { Maximize: } & \phi(\overline{\boldsymbol{x}} / \bar{T}) \\
\text { Subject to: } & \bar{y}_{l} / \bar{T} \leq c_{l} \forall l \in\{1, \ldots, L\} \\
& \pi[r] \in \mathcal{P} \forall r \in\{0,1,2, \ldots\}
\end{array}
$$

where $\phi(\gamma)$ is a given concave and entrywise non-decreasing utility function defined over $\gamma=\left(\gamma_{1}, \ldots, \gamma_{M}\right) \in \mathbb{R}^{M}$.

We note that for some problems, one may be more interested in optimizing the per-frame average $\bar{y}_{0}$, rather than the time average $\bar{y}_{0} / \bar{T}$, and such problems are treated in Section VI-A.

\section{B. Boundedness Assumptions}

We assume $x_{m}[r], T[r]$, and $y_{0}[r]$ have bounded conditional expectations, regardless of the policy. That is, there are finite constants $x_{m}^{\min }, x_{m}^{\max }, T^{\min }, T^{\max }, y_{0}^{\min }, y_{0}^{\max }$ such that for all $\pi[r] \in \mathcal{P}$ and all $m \in\{1, \ldots, M\}$ we have:

$$
\begin{aligned}
y_{0}^{\text {min }} & \leq \mathbb{E}\left\{\hat{y}_{0}(\pi[r]) \mid \pi[r]\right\} \leq y_{0}^{\max } \\
0<T^{\text {min }} & \leq \mathbb{E}\{\hat{T}(\pi[r]) \mid \pi[r]\} \leq T^{\text {max }} \\
x_{m}^{\text {min }} & \leq \mathbb{E}\left\{\hat{x}_{m}(\pi[r]) \mid \pi[r]\right\} \leq x_{m}^{\max }
\end{aligned}
$$

Define $\gamma_{m}^{\min }$ and $\gamma_{m}^{\max }$ by:

$$
\begin{aligned}
& \gamma_{m}^{\min } \triangleq \min \left[x_{m}^{\min } / T^{\min }, x_{m}^{\min } / T^{\max }\right] \\
& \gamma_{m}^{\max } \triangleq \max \left[x_{m}^{\max } / T^{\max }, x_{m}^{\max } / T^{\max }\right]
\end{aligned}
$$

Define the hyper-rectangle $\mathcal{R}$ by:

$$
\mathcal{R} \triangleq\left\{\gamma \in \mathbb{R}^{M} \mid \gamma_{m}^{\min } \leq \gamma_{m} \leq \gamma_{m}^{\max } \forall m \in\{1, \ldots, M\}\right\}
$$

Then for any algorithm that chooses policies $\pi[r] \in \mathcal{P}$ for all frames $r$, it is not difficult to show that $\bar{x}_{m}[R] / \bar{T}[R] \in \mathcal{R}$ for all $R \in\{1,2,3, \ldots\}$, where $\bar{T}[R], \bar{x}_{m}[R], \bar{T}[R]$ are frame average expectations over the first $R$ frames, as defined by (4).

Finally, we assume the conditional second moments of $T[r]$, $x_{m}[r]$, and $y_{l}[r]$ (for $l \neq 0$ ) are finite, regardless of the policy. That is, there is a finite constant $\sigma_{1}$ such that for all $\pi[r] \in \mathcal{P}$ :

$$
\begin{aligned}
\mathbb{E}\left\{\hat{T}(\pi[r])^{2} \mid \pi[r]\right\} & \leq \sigma_{1} \\
\mathbb{E}\left\{\hat{y}_{l}(\pi[r])^{2} \mid \pi[r]\right\} & \leq \sigma_{1} \forall l \in\{1, \ldots, L\} \\
\mathbb{E}\left\{\hat{x}_{m}(\pi[r])^{2} \mid \pi[r]\right\} & \leq \sigma_{1} \forall m \in\{1, \ldots, M\}
\end{aligned}
$$

\section{Optimality of i.i.d. Algorithms}

We now state the problem (5)-(7) more precisely, using lim sups which do not require existence of a well defined limit:

$$
\begin{aligned}
& \text { Minimize: } \quad \lim \sup _{R \rightarrow \infty} \frac{\bar{y}_{0}[R]}{\bar{T}[R]} \\
& \text { Subject to: } \quad \lim \sup _{R \rightarrow \infty} \frac{\bar{y}_{l}[R]}{\bar{T}[R]} \leq c_{l} \forall l \in\{1, \ldots, L\} \\
& \pi[r] \in \mathcal{P} \forall r \in\{0,1,2, \ldots\}
\end{aligned}
$$

Assume that the constraints (13)-(14) are feasible, and define ratio $^{\text {opt }}$ as the infimum ratio in (12) over all algorithms that satisfy these constraints.

Define an i.i.d. algorithm as one that, at the beginning of each new frame $r \in\{0,1,2, \ldots\}$, chooses a policy $\pi[r]$ by independently and probabilistically selecting $\pi \in \mathcal{P}$ according to some distribution that is the same for all frames $r$. Let $\pi^{*}[r]$ represent such an i.i.d. algorithm. Then the random variables $\left\{\hat{T}\left(\pi^{*}[r]\right)\right\}_{r=0}^{\infty}$ are independent and identically distributed (i.i.d.) over frames, as are $\left\{\hat{y}_{l}\left(\pi^{*}[r]\right)\right\}_{r=0}^{\infty}$. Thus, by the law of large numbers, these have well defined time averages $\bar{T}^{*}$ and $\bar{y}_{l}^{*}$ with probability 1 , where the averages are equal to the expectations over one frame.

Lemma 1: (Optimality over i.i.d. algorithms) If the constraints (13)-(14) are feasible, then for any $\delta>0$, there exists an i.i.d. algorithm $\pi^{*}[r]$ that satisfies:

$$
\begin{array}{r}
\mathbb{E}\left\{\hat{y}_{0}\left(\pi^{*}[r]\right)\right\} \leq \mathbb{E}\left\{\hat{T}\left(\pi^{*}[r]\right)\right\}\left(\text { ratio }^{o p t}+\delta\right) \\
\mathbb{E}\left\{\hat{y}_{l}\left(\pi^{*}[r]\right)\right\} \leq \mathbb{E}\left\{\hat{T}\left(\pi^{*}[r]\right)\right\}\left(c_{l}+\delta\right) \forall l \in\{1, \ldots, L\}
\end{array}
$$

Proof: See Appendix A.

\section{Optimizing Time AVERAges}

Here we develop an algorithm to treat the problem (5)-(7). To treat the constraints $\bar{y}_{l} / \bar{T} \leq c_{l}$, which are equivalent to the constraints $\bar{y}_{l} \leq c_{l} \bar{T}$, we define virtual queues $Z_{l}[r]$ for $l \in\{1, \ldots, L\}$, with finite initial condition and with update equation:

$$
Z_{l}[r+1]=\max \left[Z_{l}[r]+y_{l}[r]-c_{l} T[r], 0\right] \forall l \in\{1, \ldots, L\}
$$

The intuition is that if we can stabilize the queue $Z_{l}[r]$, then the time average of the "service process" $c_{l} T[r]$ is greater than or equal to the time average of the "arrival process" $y_{l}[r]$ (see also [12] for application to virtual power queues for meeting time average power constraints).

Let $Z[r]=\left(Z_{1}[r], \ldots, Z_{L}[r]\right)$ be the vector of virtual queues, and define the following quadratic Lyapunov function $L(\boldsymbol{Z}[r])$ :

$$
L(\boldsymbol{Z}[r]) \triangleq \frac{1}{2} \sum_{l=1}^{L} Z_{l}[r]^{2}
$$

The value $L(\boldsymbol{Z}[r])$ is a scalar measure of the size of the queue backlogs. The intuition is that if we can take actions that consistently push this value down, then queues can be stabilized. Define the frame-based conditional Lyapunov drift $\Delta(\boldsymbol{Z}[r])$ by:

$$
\Delta(\boldsymbol{Z}[r]) \triangleq \mathbb{E}\{L(\boldsymbol{Z}[r+1])-L(\boldsymbol{Z}[r]) \mid \boldsymbol{Z}[r]\}
$$


Lemma 2: Under any control decision for choosing $\pi[r] \in$ $\mathcal{P}$, we have for all $r$ and all possible $\boldsymbol{Z}[r]$ :

$$
\Delta(\boldsymbol{Z}[r]) \leq B+\mathbb{E}\left\{\sum_{l=1}^{L} Z_{l}[r]\left[y_{l}[r]-c_{l} T[r]\right] \mid \boldsymbol{Z}[r]\right\}
$$

where $B$ is a constant that satisfies for all $r$ and all possible $\boldsymbol{Z}[r]$ :

$$
B \geq \frac{1}{2} \sum_{l=1}^{L} \mathbb{E}\left\{\left(y_{l}[r]-c_{l} T[r]\right)^{2} \mid \boldsymbol{Z}[r]\right\}
$$

Such a constant $B$ exists by the boundedness assumptions in Section II-B

Proof: Squaring (17) yields:

$$
\begin{aligned}
Z_{l}[r+1]^{2} \leq & \left(Z_{l}[r]+y_{l}[r]-c_{l} T[r]\right)^{2} \\
= & Z_{l}[r]^{2}+\left(y_{l}[r]-c_{l} T[r]\right)^{2} \\
& +2 Z_{l}[r]\left(y_{l}[r]-c_{l} T[r]\right)
\end{aligned}
$$

Taking conditional expectations, dividing by 2 , and summing over $l \in\{1, \ldots, L\}$ yields the result.

\section{A. The Drift-Plus-Penalty Ratio Algorithm}

Our Drift-Plus-Penalty Ratio Algorithm is designed to minimize a sum of the variables on the right-hand-side of the drift bound (18) and a penalty term, divided by an expected frame size, as in [22]. The penalty term uses a non-negative constant $V$ that will be shown to affect a performance tradeoff:

- (Policy Selection) Every frame $r \in\{0,1,2, \ldots\}$, observe the virtual queues $\boldsymbol{Z}[r]$ and choose a policy $\pi[r] \in \mathcal{P}$ to minimize the following expression:

$$
\frac{\mathbb{E}\left\{V \hat{y}_{0}(\pi[r])+\sum_{l=1}^{L} Z_{l}[r] \hat{y}_{l}(\pi[r]) \mid \boldsymbol{Z}[r]\right\}}{\mathbb{E}\{\hat{T}(\pi[r]) \mid \boldsymbol{Z}[r]\}}
$$

- (Queue Update) Observe the resulting $\boldsymbol{y}[r]$ and $T[r]$ values, and update virtual queues $Z_{l}[r]$ by (17).

Details on minimizing (20) are given in Section D Rather than assuming we achieve the exact infimum of (20) over all policies $\pi[r] \in \mathcal{P}$, it is useful to allow our decisions to come within an additive constant $C$ of the infimum.

Definition 1: A policy $\pi[r]$ is a $C$-additive approximation for the problem (20) if for a given constant $C \geq 0$ we have:

$$
\begin{aligned}
& \frac{\mathbb{E}\left\{V \hat{y}_{0}(\pi[r])+\sum_{l=1}^{L} Z_{l}[r] \hat{y}_{l}(\pi[r]) \mid \boldsymbol{Z}[r]\right\}}{\mathbb{E}\{\hat{T}(\pi[r]) \mid \boldsymbol{Z}[r]\}} \leq \\
& C+\inf _{\pi \in \mathcal{P}}\left[\frac{\mathbb{E}\left\{V \hat{y}_{0}(\pi)+\sum_{l=1}^{L} Z_{l}[r] \hat{y}_{l}(\pi) \mid \boldsymbol{Z}[r]\right\}}{\mathbb{E}\{\hat{T}(\pi) \mid \boldsymbol{Z}[r]\}}\right]
\end{aligned}
$$

In Section $\mathrm{V}-\mathrm{B}$ it is shown that the infimum of (20) over $\pi \in \mathcal{P}$ is the same as the infimum over the extended class of probabilistically mixed strategies that choose a random $\pi \in \mathcal{P}$ according to some distribution (exactly what i.i.d. policies do every frame). Thus, if policy $\pi[r]$ is a $C$-additive approximation, then:

$$
\begin{gathered}
\mathbb{E}\left\{V \hat{y}_{0}(\pi[r])+\sum_{l=1}^{L} Z_{l}[r] \hat{y}_{l}(\pi[r]) \mid \boldsymbol{Z}[r]\right\} \leq \\
\mathbb{E}\{\hat{T}(\pi[r]) \mid \boldsymbol{Z}[r]\}\left[C+\frac{\mathbb{E}\left\{V \hat{y}_{0}\left(\pi^{*}[r]\right)+\sum_{l=1}^{L} Z_{l}[r] \hat{y}_{l}\left(\pi^{*}[r]\right)\right\}}{\mathbb{E}\left\{\hat{T}\left(\pi^{*}[r]\right)\right\}}\right](21)
\end{gathered}
$$

where $\pi^{*}[r]$ is any i.i.d. algorithm. Note that conditional expectations given $\boldsymbol{Z}[r]$ are the same as unconditional expectations under i.i.d. algorithms, because their decisions are independent of system history.

Theorem 1: (Algorithm Performance) Assume the constraints of problem (12)-(14) are feasible. Fix constants $C \geq 0$, $V \geq 0$, and assume the above algorithm is implemented using any $C$-additive approximation every frame $r$ for the minimization in 20. Assume initial conditions satisfy $\mathbb{E}\{L(\boldsymbol{Z}[0])\}<$ $\infty$. Then:

a) For all $l \in\{1, \ldots, L\}$ we have:

$$
\begin{aligned}
& \limsup _{R \rightarrow \infty} \bar{y}_{l}[R] / \bar{T}[R] \leq c_{l} \forall l \in\{1, \ldots, L\} \\
&\left.\limsup _{R \rightarrow \infty} \frac{\sum_{r=0}^{R-1} y_{l}[r]}{\sum_{r=0}^{R-1} T[r]} \leq c_{l} \quad \text { (w.p. } 1\right)
\end{aligned}
$$

where "w.p.1" stands for "with probability 1."

b) For all integers $R>0$ we have:

$$
\frac{\bar{y}_{0}[R]}{\bar{T}[R]} \leq \text { ratio }^{\text {opt }}+\frac{(B / \bar{T}[R]+C)}{V}+\frac{\mathbb{E}\{L(\boldsymbol{Z}[0])\}}{V R \bar{T}[R]}
$$

and hence:

$$
\limsup _{R \rightarrow \infty} \bar{y}_{0}[R] / \bar{T}[R] \leq \text { ratio }^{\text {opt }}+\left(B / T^{\text {min }}+C\right) / V
$$

where $B$ is defined in (19), and ratio ${ }^{o p t}$ is the optimal solution to (12)-14).

Thus, the algorithm satisfies all constraints, and the value of $V$ can be chosen appropriately large to make $\left(B / T^{\text {min }}+\right.$ $C) / V$ arbitrarily small, ensuring that the time average penalty is arbitrarily close to its optimal value ratio $^{\text {opt }}$. The tradeoff in choosing a large value of $V$ comes in the size of the $Z_{l}[r]$ queues and the number of frames required for $\mathbb{E}\left\{Z_{l}[R]\right\} / R$ to approach zero (which affects convergence time of the algorithm, see (34) in the proof). In particular, in Appendix $\mathrm{B}$ it is shown that there are constants $F_{1}, F_{2}$ such that for all $l \in\{1, \ldots, L\}$ we have:

$$
\frac{\bar{y}_{l}[R]}{\bar{T}[R]} \leq c_{l}+\frac{1}{T_{\min }} \sqrt{\frac{F_{1}+V F_{2}}{R}+\frac{\sum_{l=1}^{L} \mathbb{E}\left\{Z_{l}[0]^{2}\right\}}{R^{2}}}
$$

It is clear that the second term on the right-hand-side above vanishes as $R \rightarrow \infty$, but the number of frames required for it to be small depends on the $V$ parameter. This bound holds for general problems. A tighter bound can be obtained for problems with special structure (see (75) in Appendix G).

Proof: (Theorem 10 Consider any frame $r \in\{0,1,2, \ldots\}$. From (18) we have:

$$
\begin{gathered}
\Delta(\boldsymbol{Z}[r])+V \mathbb{E}\left\{\hat{y}_{0}(\pi[r]) \mid \boldsymbol{Z}[r]\right\} \leq B \\
+\mathbb{E}\left\{V \hat{y}_{0}(\pi[r])+\sum_{l=1}^{L} Z_{l}[r]\left[\hat{y}_{l}(\pi[r])-c_{l} \hat{T}(\pi[r])\right] \mid \boldsymbol{Z}[r]\right\}
\end{gathered}
$$

Substituting (21) into the above yields:

$$
\begin{gathered}
\Delta(\boldsymbol{Z}[r])+V \mathbb{E}\left\{\hat{y}_{0}(\pi[r]) \mid \boldsymbol{Z}[r]\right\} \leq B+ \\
\mathbb{E}\{\hat{T}(\pi[r]) \mid \boldsymbol{Z}[r]\}\left[C+\frac{\mathbb{E}\left\{V \hat{y}_{0}\left(\pi^{*}[r]\right)+\sum_{l=1}^{L} Z_{l}[r] \hat{y}_{l}\left(\pi^{*}[r]\right)\right\}}{\mathbb{E}\left\{\hat{T}\left(\pi^{*}[r]\right)\right\}}\right] \\
-\sum_{l=1}^{L} Z_{l}[r] c_{l} \mathbb{E}\{\hat{T}(\pi[r]) \mid \boldsymbol{Z}[r]\}
\end{gathered}
$$


In the above inequality, $\pi[r]$ represents the $C$-additive approximate decision actually made, and $\pi^{*}[r]$ is from any alternative i.i.d. algorithm. Fixing any $\delta>0$, plugging the i.i.d. algorithm $\pi^{*}[r]$ from (15)-(16) into the right-hand-side of (26), and letting $\delta \rightarrow 0$ yields:

$$
\begin{aligned}
& \Delta(\boldsymbol{Z}[r])+V \mathbb{E}\left\{\hat{y}_{0}(\pi[r]) \mid \boldsymbol{Z}[r]\right\} \leq B \\
& +\mathbb{E}\{\hat{T}(\pi[r]) \mid \boldsymbol{Z}[r]\}\left[C+\text { Vratio }^{\text {opt }}\right]
\end{aligned}
$$

Taking expectations of the above yields:

$$
\begin{array}{r}
\mathbb{E}\{L(\boldsymbol{Z}[r+1])\}-\mathbb{E}\{L(\boldsymbol{Z}[r])\}+V \mathbb{E}\left\{\hat{y}_{0}(\pi[r])\right\} \leq \\
B+\mathbb{E}\{\hat{T}(\pi[r])\}\left[C+\text { Vratio }^{\text {opt }}\right]
\end{array}
$$

Summing the above over $r \in\{0, \ldots, R-1\}$ for some integer $R>0$ and dividing by $R$ yields:

$$
\begin{array}{r}
\mathbb{E}\{L(\boldsymbol{Z}[R])\}-\mathbb{E}\{L(\boldsymbol{Z}[0])\} \\
R \quad V \bar{y}_{0}[R] \leq \\
B+\bar{T}[R]\left[C+\text { Vratio }^{\text {opt }}\right]
\end{array}
$$

Rearranging terms in the above and using the fact that $\mathbb{E}\{L(\boldsymbol{Z}[R])\} \geq 0$ yields the result of part (b).

To prove part (a), from (27) there is a constant $F$ such that:

$$
\Delta(\boldsymbol{Z}[r]) \leq F
$$

Thus, the drift of a quadratic Lyapunov function is bounded by a constant. Further, the second moments of per-frame changes in $Z_{l}[r]$ are bounded because of the second moment assumptions on $y_{l}[r]$ and $T[r]$. It follows that (see [25]):

$$
\begin{aligned}
\lim _{R \rightarrow \infty} \mathbb{E}\left\{Z_{l}[R]\right\} / R & =0 \\
\lim _{R \rightarrow \infty} Z_{l}[R] / R & =0 \text { (w.p.1) }
\end{aligned}
$$

Now from the queue update (17) we have for any frame $r$ :

$$
Z_{l}[r+1] \geq Z_{l}[r]+y_{l}[r]-c_{l} T[r]
$$

Summing the above over $r \in\{0, \ldots, R-1\}$ for some integer $R>0$ yields:

$$
Z_{l}[R]-Z_{l}[0] \geq \sum_{r=0}^{R-1}\left[y_{l}[r]-c_{l} T[r]\right]
$$

Taking expectations, dividing by $R$, and using $\mathbb{E}\left\{Z_{l}[0]\right\} \geq 0$ yields for all integers $R>0$ :

$$
\frac{\mathbb{E}\left\{Z_{l}[R]\right\}}{R} \geq \bar{y}_{l}[R]-c_{l} \bar{T}[R]
$$

Thus:

$$
\frac{\bar{y}_{l}[R]}{\bar{T}[R]} \leq c_{l}+\frac{\mathbb{E}\left\{Z_{l}[R]\right\}}{R \bar{T}[R]} \leq c_{l}+\frac{\mathbb{E}\left\{Z_{l}[R]\right\}}{R T^{\text {min }}}
$$

Taking limits of the above and using (31) proves (22). A similar argument uses (32) to prove (23).

Under a mild "Slater-type" assumption that ensures the constraints (13) are achievable with " $\epsilon$-slackness," the queues $Z_{l}[R]$ can be shown to be strongly stable, in the sense that the time average expectation is bounded by $O(V)$. If further mild fourth moment boundedness assumptions hold for $y_{l}[r]$ and $T[r]$ then the same bound (24) can be shown to hold for pure time averages with probability 1 (see [25] and Appendix $\mathrm{H})$.

\section{UTILITY OPTIMIZATION}

Consider now the problem (8)-(10), which seeks to maximize $\phi(\overline{\boldsymbol{x}} / \bar{T})$ subject to $\bar{y}_{l} / \bar{T} \leq c_{l}$ for all $l \in\{1, \ldots, L\}$. We transform this problem of maximizing a function of a time average ratio into a problem of the type (5)-(7). The following variation on Jensen's inequality is crucial in this transformation:

Lemma 3: (Variation on Jensen's Inequality) Let $\phi(\gamma)$ be any continuous and concave function defined over $\gamma \in \mathcal{R}$ for some closed and bounded hyper-rectangle $\mathcal{R}$.

a) Let $(T, \gamma)$ be any random vector (with an arbitrary joint distribution) that satisfies $T>0$ and $\gamma \in \mathcal{R}$ with probability 1. Assume that $0<\mathbb{E}\{T\}<\infty$. Then:

$$
\frac{\mathbb{E}\{T \phi(\gamma)\}}{\mathbb{E}\{T\}} \leq \phi\left(\frac{\mathbb{E}\{T \gamma\}}{\mathbb{E}\{T\}}\right)
$$

b) Let $(T[r], \gamma[r])$ be a sequence of arbitrarily correlated random vectors for $r \in\{0,1,2, \ldots\}$. Assume that $T[r]>0$, $\gamma[r] \in \mathcal{R}$ for all $r$ (with probability 1 ), and:

$$
0<T^{\text {min }} \leq \mathbb{E}\{T[r]\} \leq T^{\text {max }}<\infty \forall r \in\{0,1,2, \ldots\}
$$

Then for any $R>0$ :

$$
\frac{\frac{1}{R} \sum_{r=0}^{R-1} \mathbb{E}\{T[r] \phi(\gamma[r])\}}{\frac{1}{R} \sum_{r=0}^{R-1} \mathbb{E}\{T[r]\}} \leq \phi\left(\frac{\frac{1}{R} \sum_{r=0}^{R-1} \mathbb{E}\{T[r] \gamma[r]\}}{\frac{1}{R} \sum_{r=0}^{R-1} \mathbb{E}\{T[r]\}}\right)
$$

Furthermore, assuming that the limits $\overline{T \phi(\gamma)}$ and $\overline{T \gamma}$ defined below exist, we have:

$$
\overline{T \phi(\gamma)} / \bar{T} \leq \phi(\overline{T \gamma} / \bar{T})
$$

where:

$$
\begin{aligned}
\overline{T \phi(\gamma)} & \triangleq \lim _{R \rightarrow \infty} \frac{1}{R} \sum_{r=0}^{R-1} \mathbb{E}\{T[r] \phi(\gamma[r])\} \\
\overline{T \gamma} & \triangleq \lim _{R \rightarrow \infty} \frac{1}{R} \sum_{r=0}^{R-1} \mathbb{E}\{T[r] \gamma[r]\}
\end{aligned}
$$

Proof: Part (b) follows immediately from part (a) by defining the random vector $(T, \gamma)$ to be $(T[J], \gamma[J])$, where $J$ is a uniformly distributed integer in $\{0,1, \ldots, R-1\}$ that is independent of the $(T[r], \gamma[r])$ process. Part (a) is proven in Appendix E.

Now define an auxiliary vector $\gamma[r]=\left(\gamma_{1}[r], \ldots, \gamma_{M}[r]\right)$, to be chosen in the set $\mathcal{R}$ defined in (11) on every frame $r$.

Lemma 4: (Equivalent Transformation) The problem (8)(10) is equivalent to the following transformed problem:

$$
\begin{array}{cc}
\text { Maximize: } & \overline{T \phi(\gamma)} / \bar{T} \\
\text { Subject to: } & \bar{x}_{m} \geq \overline{T \gamma_{m}} \forall m \in\{1, \ldots, M\} \\
& \bar{y}_{l} / \bar{T} \leq c_{l} \forall l \in\{1, \ldots, L\} \\
& \gamma[r] \in \mathcal{R} \forall r \in\{0,1,2, \ldots\} \\
& \pi[r] \in \mathcal{P} \forall r \in\{0,1,2, \ldots\}
\end{array}
$$

Proof: We briefly sketch the proof: Let $\pi^{*}[r], \gamma^{*}[r]$ be a policy that optimally solves the above transformed problem, and assume for simplicity it yields well defined time averages $\bar{T}^{*}, \bar{y}_{l}^{*}, \bar{x}_{m}^{*}, \overline{T^{*} \phi\left(\gamma^{*}\right)}, \overline{T^{*} \gamma^{*}}$, and optimal utility 
$u t i l^{*}=\overline{T^{*} \phi\left(\gamma^{*}\right)} / \bar{T}^{*}$. Then the policy $\pi^{*}[r]$ also satisfies all constraints of problem (8)-(10), and yields:

$$
\phi\left(\overline{\boldsymbol{x}}^{*} / \bar{T}^{*}\right) \geq \phi\left(\overline{T^{*} \gamma^{*}} / \overline{T^{*}}\right) \geq \overline{T^{*} \phi\left(\gamma^{*}\right)} / \bar{T}^{*} \triangleq u t i l^{*}
$$

where the first inequality above holds by (37) and the entrywise non-decreasing property of $\phi(\gamma)$, and the second holds by (35). Thus, the optimal utility of problem (8)-(10) is greater than or equal to that of the transformed problem. A similar argument shows it is also less than or equal to the optimal utility of the transformed problem.

The transformed problem (36)-(40) has the structure of the problem (5)-(7) if we define $y_{0}[r] \triangleq-T[r] \phi(\gamma[r])$, write the constraints (37) as $\overline{T \gamma_{m}-x_{m}} \leq 0$, and define policy decision $\pi^{\prime}[r] \triangleq(\pi[r], \gamma[r]) \in \mathcal{P} \times \mathcal{R}$. The resulting algorithm is thus the same as that given in Section III-A and for this context it is given as follows: For the constraints (38), use the same virtual queues $Z_{l}[r]$ defined in (17). For the constraints (37), define virtual queues $G_{m}[r]$ for $m \in\{1, \ldots, M\}$ by:

$$
G_{m}[r+1]=\max \left[G_{m}[r]+T[r] \gamma_{m}[r]-x_{m}[r], 0\right]
$$

Define $\boldsymbol{G}[r] \triangleq\left(G_{1}[r], \ldots, G_{M}[r]\right)$. The drift-plus-penalty ratio to minimize every frame $r$ is then:

$$
\begin{aligned}
\frac{\mathbb{E}\left\{-V \hat{T}(\pi[r]) \phi(\gamma[r])+\sum_{l=1}^{L} Z_{l}[r] \hat{y}_{l}(\pi[r]) \mid \boldsymbol{Z}[r]\right\}}{\mathbb{E}\{\hat{T}(\pi[r]) \mid \boldsymbol{Z}[r]\}} \\
+\frac{\mathbb{E}\left\{\sum_{m=1}^{M} G_{m}[r]\left[\hat{T}(\pi[r]) \gamma_{m}[r]-\hat{x}_{m}(\pi[r])\right] \mid \boldsymbol{Z}[r]\right\}}{\mathbb{E}\{\hat{T}(\pi[r]) \mid \boldsymbol{Z}[r]\}}
\end{aligned}
$$

It is easy to see that the above can be minimized by separately choosing $\gamma[r] \in \mathcal{R}$ and $\pi[r] \in \mathcal{P}$ to minimize their respective terms, and that $\hat{T}(\pi[r])$ cancels out of the auxiliary variable decisions. The resulting algorithm is thus to observe $\boldsymbol{Z}[r]$ and $\boldsymbol{G}[r]$ every frame $r \in\{0,1,2, \ldots\}$ and perform the following:

- (Auxiliary Variables) Choose $\gamma[r] \in \mathcal{R}$ to maximize:

$$
V \phi(\gamma[r])-\sum_{m=1}^{M} G_{m}[r] \gamma_{m}[r]
$$

- (Policy Selection) Choose $\pi[r] \in \mathcal{P}$ to minimize:

$$
\frac{\mathbb{E}\left\{\sum_{l=1}^{L} Z_{l}[r] \hat{y}_{l}(\pi[r])-\sum_{m=1}^{M} G_{m}[r] \hat{x}_{m}(\pi[r]) \mid \boldsymbol{Z}[r]\right\}}{\mathbb{E}\{\hat{T}(\pi[r]) \mid \boldsymbol{Z}[r]\}}
$$

- (Virtual Queue Update) Update $\boldsymbol{Z}[r]$ by $(17)$ and $\boldsymbol{G}[r]$ by (41).

The auxiliary variable update is a simple deterministic maximization of a concave function over a hyper-rectangle, and can be separated into $M$ optimizations of single-variable concave functions over an interval if the utility function has the form $\phi(\gamma)=\sum_{m=1}^{M} \phi_{m}\left(\gamma_{m}\right)$. The policy selection step is again an optimization of a ratio of expectations and can be done as described in Section $\mathrm{V}$

We define a $C$-additive approximation of the above algorithm as one that, every frame $r$, chooses $\pi[r] \in \mathcal{P}$ to yield an expectation of ratios in the policy selection step that is within $C$ of the infimum. To explicitly describe the performance of the above algorithm, we write the problem (8)-(10) more precisely using limsups:

$$
\begin{array}{cc}
\text { Minimize: } & \lim \sup _{R \rightarrow \infty} \phi(\overline{\boldsymbol{x}}[R] / \bar{T}[R]) \\
\text { Subject to: } & \lim \sup _{R \rightarrow \infty} \frac{\bar{y}_{l}[R]}{\bar{T}[R]} \leq c_{l} \forall l \in\{1, \ldots, L\} \\
\pi[r] \in \mathcal{P} \forall r \in\{0,1,2, \ldots\}
\end{array}
$$

Assuming the constraints of the above problem are feasible, define $u t i l^{\text {opt }}$ as the supremum value of (42) over all algorithms that satisfy (43)-(44).

Theorem 2: Suppose the constraints of problem (42)-(44) are feasible, and a $C$-additive approximation is used every frame (for $C \geq 0$ ). Then:

a) For all $l \in\{1, \ldots, L\}$ we have:

$$
\begin{aligned}
& \limsup _{R \rightarrow \infty} \bar{y}_{l}[R] / \bar{T}[R] \leq c_{l} \forall l \in\{1, \ldots, L\} \\
& \limsup _{R \rightarrow \infty} \frac{\sum_{r=0}^{R-1} y_{l}[r]}{\sum_{r=0}^{R-1} T[r]} \leq c_{l} \quad \text { (w.p.1) }
\end{aligned}
$$

b) The achieved utility satisfies:

$$
\liminf _{R \rightarrow \infty} \phi\left(\frac{\overline{\boldsymbol{x}}[R]}{\bar{T}[R]}\right) \geq u t i l^{\text {opt }}-\frac{D}{V T^{\min }}-\frac{C}{V}
$$

where $D$ is a constant that satisfies for all $r$ and all possible $\boldsymbol{Z}[r]$ :

$$
\begin{aligned}
D \geq & \frac{1}{2} \sum_{l=1}^{L} \mathbb{E}\left\{\left[y_{l}[r]-c_{l} T[r]\right]^{2} \mid \boldsymbol{Z}[r], \boldsymbol{G}[r]\right\} \\
& +\frac{1}{2} \sum_{m=1}^{M} \mathbb{E}\left\{\left[T[r] \gamma_{m}[r]-x_{m}[r]\right]^{2} \mid \boldsymbol{Z}[r], \boldsymbol{G}[r]\right\}(45)
\end{aligned}
$$

Such a constant $D$ exists by the boundedness assumptions in Section II-B

Proof: See Appendix F.

\section{Optimizing the RATIO OF EXPECTATIONS}

Here we show how to minimize the ratio of expectations given in (20) (and also in the policy selection stage of the previous section). These problems can be written more generally as choosing a policy $\pi[r] \in \mathcal{P}$ to minimize the ratio:

$$
\frac{\mathbb{E}\{a(\pi)\}}{\mathbb{E}\{b(\pi)\}}
$$

where $a(\pi), b(\pi)$ are random functions of $\pi \in \mathcal{P}$. The function $b(\pi)$ is equal to $\hat{T}(\pi)$, and is strictly positive and satisfies the following for all $\pi \in \mathcal{P}$ :

$$
0<T^{\min } \leq \mathbb{E}\{b(\pi) \mid \pi\} \leq T^{\max }<\infty
$$

The function $a(\pi)$ depends on $\boldsymbol{Z}[r]$, and the above expectations are implicitly conditioned on $\boldsymbol{Z}[r]$, although we suppress this notation for simplicity. Define $\theta^{*}$ as the optimal ratio:

$$
\theta^{*} \triangleq \inf _{\pi \in \mathcal{P}}\left[\frac{\mathbb{E}\{a(\pi)\}}{\mathbb{E}\{b(\pi)\}}\right]
$$

If the expectation $\mathbb{E}\{b(\pi)\}$ is the same for all $\pi \in \mathcal{P}$ (such as when the frame size is independent of the policy), then $\theta^{*}$ is obtained by infimizing the numerator $\mathbb{E}\{a(\pi)\}$. This is 
typically easier (often involving learning for stochastic shortest path computations [26][5]). Otherwise, the following simple lemma is useful.

Lemma 5: We have:

$$
\inf _{\pi \in \mathcal{P}} \mathbb{E}\left\{a(\pi)-\theta^{*} b(\pi)\right\}=0
$$

Further, for any real number $\theta$, we have:

$$
\begin{array}{ll}
\inf _{\pi \in \mathcal{P}} \mathbb{E}\{a(\pi)-\theta b(\pi)\}<0 & \text { if } \theta>\theta^{*} \\
\inf _{\pi \in \mathcal{P}} \mathbb{E}\{a(\pi)-\theta b(\pi)\}>0 & \text { if } \theta<\theta^{*}
\end{array}
$$

Proof: We first assume the result of (46) and use it to prove (47)-(48). Suppose that $\theta>\theta^{*}$. We then have for any $\pi \in \mathcal{P}$ :

$$
\begin{aligned}
\mathbb{E}\{a(\pi)-\theta b(\pi)\} & =\mathbb{E}\left\{a(\pi)-\theta^{*} b(\pi)-\left(\theta-\theta^{*}\right) b(\pi)\right\} \\
& \leq \mathbb{E}\left\{a(\pi)-\theta^{*} b(\pi)\right\}-\left(\theta-\theta^{*}\right) T^{\text {min }}
\end{aligned}
$$

Thus:

$$
\begin{aligned}
\inf _{\pi \in \mathcal{P}} \mathbb{E}\{a(\pi)-\theta b(\pi)\} \leq & \inf _{\pi \in \mathcal{P}} \mathbb{E}\left\{a(\pi)-\theta^{*} b(\pi)\right\} \\
& -\left(\theta-\theta^{*}\right) T^{\text {min }} \\
= & 0-\left(\theta-\theta^{*}\right) T^{\text {min }}<0
\end{aligned}
$$

where the equality holds by (46). This proves (47).

Now suppose that $\theta<\theta^{*}$. Then for any $\pi \in \mathcal{P}$ :

$$
\begin{aligned}
\mathbb{E}\{a(\pi)-\theta b(\pi)\} & =\mathbb{E}\left\{a(\pi)-\theta^{*} b(\pi)+\left(\theta^{*}-\theta\right) b(\pi)\right\} \\
& \geq \mathbb{E}\left\{a(\pi)-\theta^{*} b(\pi)\right\}+\left(\theta^{*}-\theta\right) T^{\text {min }}
\end{aligned}
$$

Taking infimums of both sides, again using (46), proves:

$$
\inf _{\pi \in \mathcal{P}} \mathbb{E}\{a(\pi)-\theta b(\pi)\} \geq 0+\left(\theta^{*}-\theta\right) T^{\text {min }}>0
$$

This proves (48).

It remains only to prove (46). We have for any policy $\pi \in \mathcal{P}$ :

$$
\frac{\mathbb{E}\{a(\pi)\}}{\mathbb{E}\{b(\pi)\}} \geq \inf _{\pi \in \mathcal{P}}\left[\frac{\mathbb{E}\{a(\pi)\}}{\mathbb{E}\{b(\pi)\}}\right] \triangleq \theta^{*}
$$

Therefore, because $\mathbb{E}\{b(\pi)\}>0$, we have for all $\pi \in \mathcal{P}$ :

$$
\mathbb{E}\{a(\pi)\}-\theta^{*} \mathbb{E}\{b(\pi)\} \geq 0
$$

and hence:

$$
\inf _{\pi \in \mathcal{P}} \mathbb{E}\left\{a(\pi)-\theta^{*} b(\pi)\right\} \geq 0
$$

It remains only to prove the reverse inequality. Fix $\delta>0$. By definition of $\theta^{*}$ as the infimum ratio, there is a policy $\pi^{*} \in \mathcal{P}$ that satisfies:

$$
\frac{\mathbb{E}\left\{a\left(\pi^{*}\right)\right\}}{\mathbb{E}\left\{b\left(\pi^{*}\right)\right\}} \leq \theta^{*}+\delta
$$

Thus:

$$
\mathbb{E}\left\{a\left(\pi^{*}\right)\right\} \leq \theta^{*} \mathbb{E}\left\{b\left(\pi^{*}\right)\right\}+\delta \mathbb{E}\left\{b\left(\pi^{*}\right)\right\}
$$

and so:

$$
\mathbb{E}\left\{a\left(\pi^{*}\right)\right\}-\theta^{*} \mathbb{E}\left\{b\left(\pi^{*}\right)\right\} \leq \delta T^{\max }
$$

Because $\pi^{*}$ is just a particular algorithm in $\mathcal{P}$, it follows that:

$$
\inf _{\pi \in \mathcal{P}} \mathbb{E}\left\{a(\pi)-\theta^{*} b(\pi)\right\} \leq \delta T^{\max }
$$

This holds for all $\delta>0$. Taking a limit as $\delta \rightarrow 0$ completes the proof.

\section{A. The Bisection Algorithm}

Lemma 5 immediately leads to the following simple bisection algorithm: Suppose we have upper and lower bounds $\theta_{\min }$ and $\theta_{\max }$, so that we know $\theta_{\min } \leq \theta^{*} \leq \theta_{\max }$. Then we can define $\theta=\left(\theta_{\min }+\theta_{\max }\right) / 2$, and compute the value of $\inf _{\pi \in \mathcal{P}} \mathbb{E}\{a(\pi)-\theta b(\pi)\}$. If the result is 0 , then $\theta=\theta^{*}$. If positive, then $\theta<\theta^{*}$, and otherwise $\theta>\theta^{*}$. We can then refine our upper and lower bounds. This leads to a simple iterative algorithm where the distance between the upper and lower bounds decreases by a factor of 2 on each iteration. It thus approaches the optimal $\theta^{*}$ value exponentially fast. Each step of the iteration involves minimizing an expectation, rather than a ratio of expectations.

\section{B. Optimizing over Pure Policies}

Note that for any set of policies $\mathcal{S}$, Lemma 5 implies that $\inf _{\pi \in \mathcal{S}} \mathbb{E}\{a(\pi)-\theta b(\pi)\}=0$ if and only if $\theta=$ $\inf _{\pi \in \mathcal{S}} \mathbb{E}\{a(\pi)\} / \mathbb{E}\{b(\pi)\}$. Now suppose we have a set of policies $\mathcal{P}^{\text {pure }}$ that we call pure policies, and that the policy space $\mathcal{P}$ consists of all pure policies as well as all "mixtures" (or convex combinations) of pure policies, being policies that choose a pure policy in $\mathcal{P}^{\text {pure }}$ with some particular probability distribution. More generally, define $\Omega$ as the set of all vectors $(\mathbb{E}\{a(\pi)\}, \mathbb{E}\{b(\pi)\})$ achievable over $\pi \in \mathcal{P}^{\text {pure }}$, and suppose the set of all $(\mathbb{E}\{a(\pi)\}, \mathbb{E}\{b(\pi)\})$ achievable over $\pi \in \mathcal{P}$ is equal to the convex hull of $\Omega$. Recall that $\theta^{*}$ is the infimum ratio of $\mathbb{E}\{a(\pi)\} / \mathbb{E}\{b(\pi)\}$ over $\pi \in \mathcal{P}$. Then:

$$
\begin{aligned}
0=\inf _{\pi \in \mathcal{P}} \mathbb{E}\left\{a(\pi)-\theta^{*} b(\pi)\right\} & =\inf _{(a, b) \in \operatorname{Conv}(\Omega)}\left[a-\theta^{*} b\right] \\
& =\inf _{(a, b) \in \Omega}\left[a-\theta^{*} b\right] \\
& =\inf _{\pi \in \mathcal{P}^{\text {pure }}} \mathbb{E}\left\{a(\pi)-\theta^{*} b(\pi)\right\}
\end{aligned}
$$

where the third inequality holds because the infimum of a linear function over the convex hull of a set is equal to the infimum over the set itself. It follows that $\theta^{*}$ is also the infimum ratio of $\mathbb{E}\{a(\pi)\} / \mathbb{E}\{b(\pi)\}$ over $\pi \in \mathcal{P}^{\text {pure }}$.

This means that to achieve the infimum ratio over policies $\pi \in \mathcal{P}$, it suffices to restrict our search to pure policies.

\section{Optimizing with Initial Information}

Suppose at the beginning of each frame, we observe a vector $\boldsymbol{\eta}[r]$ of initial information that can affect the penalties and frame size. Suppose that $\{\boldsymbol{\eta}[r]\}_{r=0}^{\infty}$ is i.i.d. over frames. Each policy $\pi \in \mathcal{P}$ first observes $\boldsymbol{\eta}[r]$ and then chooses a sub-policy $\pi^{\prime} \in \mathcal{P}_{\boldsymbol{\eta}[r]}$, where $\mathcal{P}_{\boldsymbol{\eta}[r]}$ is a space that possibly depends on $\boldsymbol{\eta}[r]$. To minimize $\mathbb{E}\{a(\pi)\}$, it suffices to observe $\boldsymbol{\eta}[r]$ and choose $\pi^{\prime} \in \mathcal{P}_{\boldsymbol{\eta}[r]}$ to minimize the conditional expectation $\mathbb{E}\left\{a\left(\pi^{\prime}\right) \mid \boldsymbol{\eta}[r]\right\}$. However, this is not necessarily true for minimizing the ratio $\mathbb{E}\{a(\pi)\} / \mathbb{E}\{b(\pi)\}$.

A correct approach is the following: If $\theta^{*}$ is known, we can simply choose $\pi^{\prime} \in \mathcal{P}_{\boldsymbol{\eta}[r]}$ to minimize:

$$
\mathbb{E}\left\{a\left(\pi^{\prime}\right)-\theta^{*} b\left(\pi^{\prime}\right) \mid \boldsymbol{\eta}[r]\right\}
$$


If $\theta^{*}$ is unknown, we can carry out the bisection routine. Let $\theta$ be the midpoint in the current iteration. We must compute:

$$
\inf _{\pi \in \mathcal{P}} \mathbb{E}\{a(\pi)-\theta b(\pi)\}=\mathbb{E}\left\{\inf _{\pi^{\prime} \in \mathcal{P}_{\boldsymbol{\eta}[\boldsymbol{r}]}} \mathbb{E}\left\{a\left(\pi^{\prime}\right)-\theta b\left(\pi^{\prime}\right) \mid \boldsymbol{\eta}[r]\right\}\right\}
$$

The infimizing decision $\pi^{\prime}$ can be made by observing $\boldsymbol{\eta}[r]$, without requiring knowledge of its probability distribution. However, the value in (49) cannot be computed without knowledge of this distribution. Instead, suppose we have $W$ i.i.d. samples $\left\{\boldsymbol{\eta}_{w}\right\}_{w=1}^{W}$. We can then approximate the value in (49) by the function $\operatorname{val}(\theta)$ defined below:

$$
\operatorname{val}(\theta) \triangleq \frac{1}{W} \sum_{w=1}^{W} \inf _{\pi^{\prime} \in \mathcal{P}_{\boldsymbol{\eta}_{w}}} \mathbb{E}\left\{a\left(\pi^{\prime}\right)-\theta b\left(\pi^{\prime}\right) \mid \boldsymbol{\eta}_{w}\right\}
$$

By the law of large numbers, $\operatorname{val}(\theta)$ approaches the exact value of (49) with a large choice of $W$. The bisection routine can be carried out using the $\operatorname{val}(\theta)$ approximation, being sure to use the same samples at each step of the iteration (but different samples on each frame $r$ ). Note that $\operatorname{val}(\theta)$ is nonincreasing in $\theta$, so the bisection will converge provided that it is initialized so that $\operatorname{val}\left(\theta_{\min }\right) \geq 0$ and $\operatorname{val}\left(\theta_{\max }\right) \leq 0$. If we cannot independently generate $W$ samples, we use the $W$ past observed values of $\boldsymbol{\eta}[r]$ from previous frames. There is a subtle issue here, as these past values have influenced system performance and are thus correlated with the current $a(\pi)$ and $b(\pi)$ functions. However, a delayed queue argument similar to that given in [27] shows these past values can still be used.

\section{Alternative Algorithms without Ratio MINIMIZATION}

We first present an alternative formulation to (5)-(7) that is easier and does not require minimizing a ratio of expectations every slot. We then present an alternative algorithm to the original problem (5)-(7) that does not require a ratio of minimizations, but which yields a less explicit convergence result.

\section{A. Alternative Formulation}

Note that constraints of the form $\bar{y}_{l} \leq 0$ are equivalent to $\bar{y}_{l} / \bar{T} \leq c_{l}$ in the special case $c_{l}=0$, and thus can be handled using the framework of this paper. Thus, if $f[r]$ is some penalty, and if we desire the constraint $\bar{f} \leq 6.7$, then we can define $y[r] \triangleq f[r]-6.7$ and note that the desired constraint is equivalent to $\bar{y} \leq 0$. In other words, constraints of the form $\bar{y}_{l} / \bar{T} \leq c_{l}$ are more general than constraints of the form $\bar{y}_{l} \leq 0$ or $\bar{y}_{l} \leq c$ for some constant $c$, and contain these as special cases.

Now consider the following problem structure:

$$
\begin{array}{cc}
\text { Minimize: } & \bar{y}_{0} \\
\text { Subject to: } & \bar{y}_{l} / \bar{T} \leq c_{l} \forall l \in\{1, \ldots, L\} \\
& \pi[r] \in \mathcal{P} \forall r \in\{0,1,2, \ldots\}
\end{array}
$$

Such a problem has a different structure than the problem (5)(7), and is easier to solve as it does not require a ratio of expectations. It can be solved using the same virtual queues
$Z_{l}[r]$ in (17), but every frame $r$ observing $\boldsymbol{Z}[r]$ and selecting a policy $\pi[r] \in \mathcal{P}$ to minimize the following expression:

$$
\mathbb{E}\left\{V \hat{y}_{0}(\pi[r])+\sum_{l=1}^{L} Z_{l}[r]\left[\hat{y}_{l}(\pi[r])-c_{l} \hat{T}(\pi[r])\right] \mid \boldsymbol{Z}[r]\right\}
$$

Analysis of this algorithm is given in Appendix C.

\section{B. Alternative Algorithm}

The following is an alternative algorithm for the original problem (5)-(7) that does not require a ratio minimization (and hence does not require a bisection step): Use the same virtual queues $Z_{l}[r]$ in (17). Define $\theta[0]=0$, and define $\theta[R]$ for $R \in\{1,2,3, \ldots\}$ by:

$$
\theta[R] \triangleq \sum_{r=0}^{R-1} y_{0}[r] / \sum_{r=0}^{R-1} T[r]
$$

Every frame $r$, observe $Z[r]$ and $\theta[r]$ and select a policy $\pi[r] \in$ $\mathcal{P}$ to minimize the following expression:

$$
\begin{gathered}
\mathbb{E}\left\{V\left[\hat{y}_{0}(\pi[r])-\theta[r] \hat{T}(\pi[r])\right] \mid \boldsymbol{Z}[r], \theta[r]\right\} \\
+\mathbb{E}\left\{\sum_{l=1}^{L} Z_{l}[r]\left[\hat{y}_{l}(\pi[r])-c_{l} \hat{T}(\pi[r])\right] \mid \boldsymbol{Z}[r], \theta[r]\right\}
\end{gathered}
$$

It is shown in Appendix D that all constraints are met, and that if $\theta[r]$ converges to a constant with probability 1 , then with probability 1 :

$$
\lim _{R \rightarrow \infty} \sum_{r=0}^{R-1} y_{0}[r] / \sum_{r=0}^{R-1} T[r] \leq \text { ratio }^{\text {opt }}+O(1 / V)
$$

The disadvantage is that the convergence time is not as clear as that given in part (b) of Theorem 1 Further, use of the time average (51) makes it difficult to adapt to changes in system parameters, so that it may be better to approximate (51) with a moving average or an exponentially decaying average.

\section{Simulations for a TAsk Processing Network}

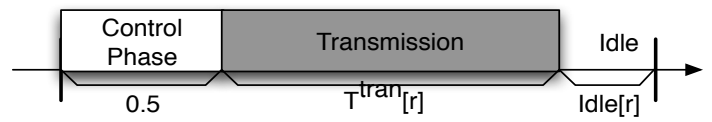

Fig. 2. An illustration of the 3 phases of a renewal frame $r \in\{0,1,2, \ldots\}$.

Here we provide a simple task processing example. An infinite sequence of tasks must be processed one at a time with the help of a network of 5 wireless devices. This applies, for example, in scenarios similar to [23] where each new task represents an event that is sensed by the wireless devices (each at different sensing qualities [28]), and we must select which device reports the event information. The renewal structure is shown in Fig. 2. At the beginning of each new task $r$, a period of 0.5 time units is expended to communicate control information about the task. Each of the 5 devices expends 0.5 units of energy in this control phase. At the end of this phase, the network controller obtains a vector $\boldsymbol{\eta}[r]$ of parameters for task $r$. The vector $\boldsymbol{\eta}[r]$ has the form:

$$
\boldsymbol{\eta}[r]=\left[\left(\text { qual }_{1}[r], T_{1}^{\text {tran }}[r]\right), \ldots,\left(q u a l_{5}[r], T_{5}^{\text {tran }}[r]\right)\right]
$$

where for each $l \in\{1, \ldots, 5\}$, qual $_{l}[r]$ is a real number representing the information quality if device $l$ is chosen to process task $r$, and $T_{l}^{\operatorname{tran}}[r]$ is the transmission time required 


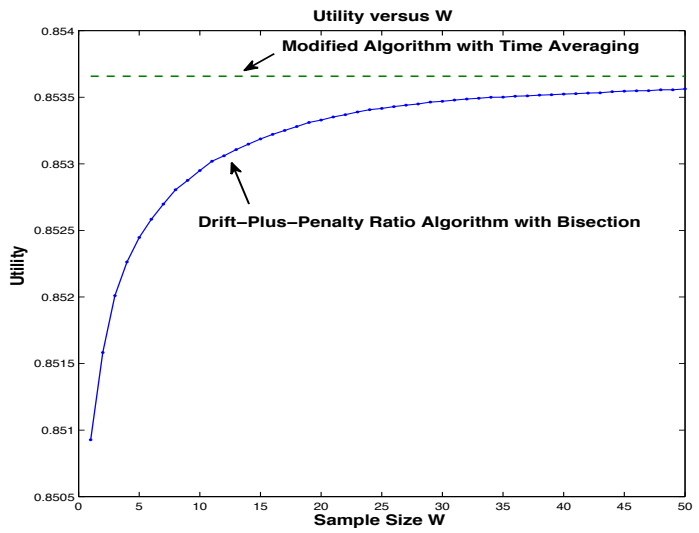

Fig. 3. Utility for the drift-plus-penalty ratio algorithm (with bisection) and the time-averaged alternative.

for device $l$ to transmit the corresponding information to a receiving station. The controller must choose one of the 5 devices to process the task, and must also choose the amount of idle time at the end of the frame (chosen within the interval $\left[0, I^{\max }\right]$ for some constant $I^{\max }>0$ ), so that the policy decision $\pi[r]$ has the form:

$\pi[r]=(l[r], I d l e[r]) \in\{1,2,3,4,5\} \times\left\{I \in \mathbb{R} \mid 0 \leq I \leq I^{\max }\right\}$

Define $P^{\text {tran }}$ as the power expenditure associated with wireless transmission. The chosen device $l[r]$ expends $P^{\text {tran }} \times$ $T_{l[r]}^{t r a n}$ units of energy in the transmit phase, while all other devices $l \neq l[r]$ expend no energy in this phase. None of the devices expend energy in the idle phase, which helps to limit the average power expenditure in the system.

The goal is to maximize the quality of information (q.o.i) per unit time subject to an average power constraint of 0.25 at each device. Define $\hat{y}_{0}(\pi[r])$ as -1 times the q.o.i. obtained for task $r, \hat{y}_{l}(\pi[r])$ as the energy expended by device $l$ on task $r$, and $\hat{T}(\pi[r])$ as the frame duration for task $r$ :

$$
\begin{aligned}
& \hat{y}_{0}(\pi[r]) \triangleq-\text { qual }_{l[r]}[r] \\
& \hat{y}_{l}(\pi[r]) \triangleq 0.5+P^{\operatorname{tran}} T_{l}^{\operatorname{tran}}[r] 1_{\{l[r]=l\}} \quad \forall l \in\{1, \ldots, 5\} \\
& \hat{T}(\pi[r]) \triangleq 0.5+T_{l[r]}^{\operatorname{tran}}[r]+\operatorname{Idle}[r]
\end{aligned}
$$

where $1_{\{l[r]=l\}}$ is an indicator function that is 1 if $l[r]=l$ and 0 else. The problem is then to minimize $\bar{y}_{0} / \bar{T}$ subject to $\bar{y}_{l} / \bar{T} \leq 0.25$ for all $l \in\{1, \ldots, 5\}$.

We simulate the drift-plus-penalty ratio algorithm for $10^{6}$ frames, using the bisection method with $W$ past samples of $\boldsymbol{\eta}[r]$ as in (50) of Section $\mathrm{V}-\mathrm{C}$ We use $P^{\text {tran }}=1.0$, $I^{\max }=5.0$. The vectors $\{\boldsymbol{\eta}[r]\}_{r=0}^{\infty}$ are assumed to be i.i.d. with independently chosen components, where $T_{l}^{\operatorname{tran}}[r]$ is uniformly distributed in $[0.5,2.5]$ for all $l$, and qual $_{l}[r]$ is uniformly distributed in $[0, l]$ for $l \in\{1,2,3,4,5\}$ (so that device 5 tends to have the highest quality, while device 1 tends to have the lowest). We initialize $\theta_{\min }=-5 V, \theta_{\max } \triangleq \sum_{l=1}^{5} Z_{l}[r] 3$. Each step of the bisection computes $\operatorname{val}(\theta)$ in (50) according to a simple deterministic optimization. In particular, for the $w$ th term in $\operatorname{val}(\theta)$, corresponding to sample $\boldsymbol{\eta}_{w}$, we choose Idle $[r, w]=0$ whenever $\theta \leq 0$, and Idle $[r, w]=I^{\max }$ if $\theta>0$, and choose $l[r, w]$ as the index $l \in\{1, \ldots, 5\}$ that minimizes:

$$
-\operatorname{Vqual}_{l}[r, w]+\left(Z_{l}[r] P^{\text {tran }}-\theta\right) T_{l}^{\text {tran }}[r, w]
$$

The bisection routine is run for each frame until $\theta_{\max }-$ $\theta_{\text {min }}<0.001$. Using $V=100$, the resulting q.o.i per unit time is plotted in Fig. 3. This increases to its optimal value as $W$ is increased. However, in this example, $W$ does not need to be very large for accurate results: Even $W=1$ produces a value that is near optimal (note that the $y$-axis in Fig. 3 distinguishes utility only in the 3rd significant digit).

All average power constraints are met in all simulations (for each $W$ ). Results for $W=10$ are: q.o. $i \bar{T}=0.852950$, $\bar{T}=3.180275, \overline{I d l e}=1.421260, \bar{y}_{0}=-2.712615$, and:

$$
\begin{gathered}
\bar{y}_{1} / \bar{T}=0.182335 \leq 0.25 \\
\bar{y}_{2} / \bar{T}=0.249547 \leq 0.25, \bar{y}_{3} / \bar{T}=0.250018 \leq 0.25 \\
\bar{y}_{4} / \bar{T}=0.250032 \leq 0.25, \bar{y}_{5} / \bar{T}=0.250046 \leq 0.25
\end{gathered}
$$

It can be seen that devices $\{2, \ldots, 5\}$ are utilized to their maximum power constraints because these tend to give the highest quality, while average power for device 1 is slack.

The alternative algorithm of Section VI-B, which does not require a bisection routine and amounts to a simple deterministic optimization for 52 every frame, achieves similar time average power expenditures to the above. It also achieves utility as shown in Fig. 3, being the constant that does not depend on $W$ (as no sampling from the past is needed). Its utility is slightly larger than that of the bisection algorithm, and is approached by the bisection algorithm as $W$ increases. It appears that this algorithm is simpler and yields "automatic learning" by using the time average value $\theta[r]$, but it might have trouble adapting if system parameters change.

Details on the particular decisions made in the simulation on each frame are provided in Appendix G. There, it is also shown that the particular structure of this example admits deterministic bounds on the constraint violations. In particular, if $I^{\max }$ is chosen to be suitably large (11.0 in this case), then we can guarantee that for all integers $R>0$ :

$$
\frac{\sum_{r=0}^{R-1} y_{l}[r]}{\sum_{r=0}^{R-1} T[r]} \leq c_{l}+\frac{d_{1}+d_{2} V}{R}
$$

for some positive constants $d_{1}, d_{2}$. If we re-run the simulations using $I^{\max }=11.0$ and $W=10$, we get similar average values as given above, and in particular the algorithm results in the same $\overline{I d l e} \approx 1.42$, as expected.

\section{CONCLUSION}

We have developed a method for optimizing time averages in general renewal systems. Every renewal frame, a policy is chosen that affects the frame size and also affects a penalty vector and/or an attribute vector. A dynamic algorithm was developed to minimize the time average of one penalty subject to time average constraints on the others. A related algorithm was developed to maximize a concave function of the time average attribute vector, subject to time average constraints. This work extends the theory of Lyapunov optimization to treat much more general classes of systems, including task processing networks with variable length scheduling modes. 


\section{ApPendix A - Proof of Lemma 1}

We present a definition and a simple lemma before the proof of Lemma 11. Define $\Gamma$ as the set of all vectors $\left[y_{0}, y_{1}, \ldots, y_{L}, T\right] \in \mathbb{R}^{L+2}$ that are achievable as time averages under i.i.d. algorithms. Thus, a vector $\left[y_{0}, y_{1}, \ldots, y_{L}, T\right]$ is in the set $\Gamma$ if and only if there is an i.i.d. algorithm $\pi^{*}[r]$ such that:

$$
\begin{aligned}
\mathbb{E}\left\{\hat{y}_{l}\left(\pi^{*}[r]\right)\right\} & =y_{l} \forall l \in\{0,1, \ldots, L\} \\
\mathbb{E}\left\{\hat{T}\left(\pi^{*}[r]\right)\right\} & =T
\end{aligned}
$$

It is easy to show that the set $\Gamma$ is bounded and convex. Further, for any frame $r$ and under any (possibly non-i.i.d.) algorithm $\pi[r]$, we have:

$$
\mathbb{E}\left\{\left[\hat{y}_{0}(\pi[r]), \ldots, \hat{y}_{L}(\pi[r]), \hat{T}(\pi[r])\right]\right\} \in \Gamma
$$

This is because the policy chooses $\pi[r]$ according to some conditional distribution given the past history, but this distribution can be viewed on frame $r$ as one that is from an i.i.d. algorithm.

Lemma 6: For any algorithm that chooses $\pi[r] \in \mathcal{P}$ for all frames $r$, we have for all integers $R>0$ :

$$
\frac{1}{R} \sum_{r=0}^{R-1} \mathbb{E}\left\{\left[\hat{y}_{0}(\pi[r]), \ldots, \hat{y}_{L}(\pi[r]), \hat{T}(\pi[r])\right]\right\} \in \Gamma
$$

Proof: (Lemma 6) Each of the individual terms in the sum is in $\Gamma$, and so the average of these terms is in $\Gamma$ (because $\Gamma$ is convex).

We now prove Lemma 1 Suppose the constraints of problem (12)-(14) are feasible, and define ratio ${ }^{o p t}$ as the infimum of the objective function over all feasible algorithms. Fix any $\delta>0$. Then there must be an algorithm $\pi[r]$ that satisfies the constraints (12)-14 and yields a limsup ratio within $\delta / 2$ of the infimum. That is, $\pi[r] \in \mathcal{P}$ for all frames $r$, and:

$$
\begin{aligned}
& \limsup _{R \rightarrow \infty}\left[\frac{\frac{1}{R} \sum_{r=0}^{R-1} \mathbb{E}\left\{\hat{y}_{0}(\pi[r])\right\}}{\frac{1}{R} \sum_{r=0}^{R-1} \mathbb{E}\{\hat{T}(\pi[r])\}}\right] \leq \text { ratio }^{\text {opt }}+\delta / 2 \\
& \limsup _{R \rightarrow \infty}\left[\frac{\frac{1}{R} \sum_{r=0}^{R-1} \mathbb{E}\left\{\hat{y}_{l}(\pi[r])\right\}}{\frac{1}{R} \sum_{r=0}^{R-1} \mathbb{E}\{\hat{T}(\pi[r])\}}\right] \leq c_{l}, \forall l \in\{1, \ldots, L\}
\end{aligned}
$$

It follows that there is a finite integer $R^{*}$ such that:

$$
\begin{aligned}
& {\left[\frac{\frac{1}{R^{*}} \sum_{r=0}^{R^{*}-1} \mathbb{E}\left\{\hat{y}_{0}(\pi[r])\right\}}{\frac{1}{R^{*}} \sum_{r=0}^{R^{*}-1} \mathbb{E}\{\hat{T}(\pi[r])\}}\right] \leq \text { ratio }^{\text {opt }}+\delta} \\
& {\left[\frac{\frac{1}{R^{*}} \sum_{r=0}^{R^{*}-1} \mathbb{E}\left\{\hat{y}_{l}(\pi[r])\right\}}{\frac{1}{R^{*}} \sum_{r=0}^{R^{*}-1} \mathbb{E}\{\hat{T}(\pi[r])\}}\right] \leq c_{l}+\delta, \quad \forall l \in\{1, \ldots, L\}}
\end{aligned}
$$

By Lemma 6, we know there is an i.i.d. algorithm $\pi^{*}[r]$ such that:

$$
\begin{array}{r}
\frac{1}{R^{*}} \sum_{r=0}^{R^{*}-1} \mathbb{E}\left\{\left[\hat{y}_{0}(\pi[r]), \ldots, \hat{y}_{L}(\pi[r]), \hat{T}(\pi[r])\right]\right\}= \\
\mathbb{E}\left\{\left[\hat{y}_{0}\left(\pi^{*}[r]\right), \ldots, \hat{y}_{L}\left(\pi^{*}[r]\right), \hat{T}\left(\pi^{*}[r]\right)\right]\right\}
\end{array}
$$

Plugging this identity into the above inequalities yields:

$$
\begin{aligned}
& \frac{\mathbb{E}\left\{\hat{y}_{0}\left(\pi^{*}[r]\right)\right\}}{\mathbb{E}\left\{\hat{T}\left(\pi^{*}[r]\right)\right\}} \leq \text { ratio }^{\text {opt }}+\delta \\
& \frac{\mathbb{E}\left\{\hat{y}_{l}\left(\pi^{*}[r]\right)\right\}}{\mathbb{E}\left\{\hat{T}\left(\pi^{*}[r]\right)\right\}} \leq c_{l}+\delta, \quad \forall l \in\{1, \ldots, L\}
\end{aligned}
$$

Multiplying the above by the positive number $\mathbb{E}\left\{\hat{T}\left(\pi^{*}[r]\right)\right\}$ proves Lemma 1

\section{APPENDix B - Bound on $\bar{y}_{l}[R] / \bar{T}[R]$ IN TheOREm 1}

This section provides an upper bound on $\bar{y}_{l}[R] / \bar{T}[R]$, which shows how long is required to come close to meeting the constraints $\bar{y}_{l} / \bar{T} \leq c_{l}$. Suppose the assumptions of Theorem 1 hold. From (27) we have for all frames $r$ :

$$
\Delta(\boldsymbol{Z}[r]) \leq B+T^{\max }\left[C+\text { Vratio }^{\text {opt }}\right]-V y_{0}^{\min }
$$

where we have used the fact that the conditional expectation of $\hat{y}_{0}(\pi[r])$ is bounded below by $y_{0}^{\min }$, and the conditional expectation of $\hat{T}(\pi[r])$ is bounded above by $T^{\max }$. It follows that:

$$
\Delta(\boldsymbol{Z}[r]) \leq\left(F_{1}+V F_{2}\right) / 2
$$

where the constants $F_{1}$ and $F_{2}$ are defined:

$$
F_{1} \triangleq 2\left(B+T^{\max } C\right), F_{2} \triangleq 2\left(T^{\max } \text { ratio }^{\text {opt }}-y_{0}^{\min }\right)
$$

Substituting the definition of $\Delta(\boldsymbol{Z}[r])$ in (53) yields:

$$
\mathbb{E}\{L(\boldsymbol{Z}[r+1])-L(\boldsymbol{Z}[r]) \mid \boldsymbol{Z}[r]\} \leq\left(F_{1}+V F_{2}\right) / 2
$$

Taking expectations of the above and using the law of iterated expectations yields:

$$
\mathbb{E}\{L(\boldsymbol{Z}[r+1])\}-\mathbb{E}\{L(\boldsymbol{Z}[r])\} \leq\left(F_{1}+V F_{2}\right) / 2
$$

The above holds for all frames $r$. Summing over $r \in$ $\{0,1, \ldots, R-1\}$ (for some positive integer $R$ ) and dividing by $R$ gives:

$$
\frac{\mathbb{E}\{L(\boldsymbol{Z}[R])\}-\mathbb{E}\{L(\boldsymbol{Z}[0])\}}{R} \leq\left(F_{1}+V F_{2}\right) / 2
$$

Using the fact that $L(\boldsymbol{Z}[r]) \triangleq \frac{1}{2} \sum_{l=1}^{L} Z_{l}[r]^{2}$, we have:

$$
\sum_{l=1}^{L} \frac{\mathbb{E}\left\{Z_{l}[R]^{2}\right\}}{R} \leq\left(F_{1}+V F_{2}\right)+\frac{\sum_{l=1}^{L} \mathbb{E}\left\{Z_{l}[0]^{2}\right\}}{R}
$$

Thus, for every $l \in\{1, \ldots, L\}$ we have:

$$
\frac{\mathbb{E}\left\{Z_{l}[R]^{2}\right\}}{R^{2}} \leq \frac{F_{1}+V F_{2}}{R}+\frac{\sum_{l=1}^{L} \mathbb{E}\left\{Z_{l}[0]^{2}\right\}}{R^{2}}
$$

By Jensen's inequality, $\mathbb{E}\left\{Z_{l}[R]\right\}^{2} \leq \mathbb{E}\left\{Z_{l}[R]^{2}\right\}$, and so for all integers $R>0$ and all $l \in\{1, \ldots, L\}$ we have:

$$
\frac{\mathbb{E}\left\{Z_{l}[R]\right\}}{R} \leq \sqrt{\frac{F_{1}+V F_{2}}{R}+\frac{\sum_{l=1}^{L} \mathbb{E}\left\{Z_{l}[0]^{2}\right\}}{R^{2}}}
$$

Therefore, from (34) we have for all $l \in\{1, \ldots, L\}$ :

$$
\begin{aligned}
\frac{\bar{y}_{l}[R]}{\bar{T}[R]} & \leq c_{l}+\frac{1}{T_{\min }} \frac{\mathbb{E}\left\{Z_{l}[R]\right\}}{R} \\
& \leq c_{l}+\frac{1}{T_{\min }} \sqrt{\frac{F_{1}+V F_{2}}{R}+\frac{\sum_{l=1}^{L} \mathbb{E}\left\{Z_{l}[0]^{2}\right\}}{R^{2}}}
\end{aligned}
$$




\section{APPENDiX C - ANALYSis of Alternative FORMULATION}

Consider the alternative problem from Section VI-A

$$
\begin{aligned}
& \text { Minimize: } \quad \lim \sup _{R \rightarrow \infty} \bar{y}_{0}[R] \\
& \text { Subject to: } \quad \lim \sup _{R \rightarrow \infty} \frac{\bar{y}_{l}[R]}{\bar{T}[R]} \leq c_{l} \forall l \in\{1, \ldots, L\} \\
& \pi[r] \in \mathcal{P} \forall r \in\{0,1,2, \ldots\}
\end{aligned}
$$

Assume the same boundedness assumptions of Section $\amalg-\mathrm{B}$ hold. Further assume that a $C$-additive approximation of the algorithm of Section VI-A is implemented, so that every frame $r$ we observe $\boldsymbol{Z}[r]$ and choose $\pi[r] \in \mathcal{P}$ to yield:

$$
\begin{aligned}
& \mathbb{E}\left\{V \hat{y}_{0}(\pi[r])+\sum_{l=1}^{L} Z_{l}[r]\left[\hat{y}_{l}(\pi[r])-c_{l} \hat{T}(\pi[r])\right] \mid \boldsymbol{Z}[r]\right\} \leq \\
& C+\mathbb{E}\left\{V \hat{y}_{0}\left(\pi^{*}[r]\right)+\sum_{l=1}^{L} Z_{l}[r]\left[\hat{y}_{l}\left(\pi^{*}[r]\right)-c_{l} \hat{T}\left(\pi^{*}[r]\right)\right]\right\}
\end{aligned}
$$

where $C$ is a given non-negative constant, and $\pi^{*}[r]$ is any i.i.d. algorithm.

Theorem 3: Under the above assumptions, and assuming the constraints of the problem (54)-(56) are feasible, then:

a) For all $l \in\{1, \ldots, L\}$ we have:

$$
\begin{aligned}
& \limsup _{R \rightarrow \infty} \bar{y}_{l}[R] / \bar{T}[R] \leq c_{l} \forall l \in\{1, \ldots, L\} \\
& \limsup _{R \rightarrow \infty} \frac{\sum_{r=0}^{R-1} y_{l}[r]}{\sum_{r=0}^{R-1} T[r]} \leq c_{l} \text { (w.p.1) }
\end{aligned}
$$

b) For all integers $R>0$ we have:

$$
\bar{y}_{0}[R] \leq y_{0}^{o p t}+\frac{B+C}{V}+\frac{\mathbb{E}\{L(\boldsymbol{Z}[0])\}}{V R}
$$

where $B$ is defined in (19), and $y_{0}^{o p t}$ is the infimum value of (54) subject to (55)-(56).

Proof: Consider any frame $r \in\{0,1,2, \ldots\}$. From (18) we have:

$$
\begin{gathered}
\Delta(\boldsymbol{Z}[r])+V \mathbb{E}\left\{\hat{y}_{0}(\pi[r]) \mid \boldsymbol{Z}[r]\right\} \leq B \\
+\mathbb{E}\left\{V \hat{y}_{0}(\pi[r])+\sum_{l=1}^{L} Z_{l}[r]\left[\hat{y}_{l}(\pi[r])-c_{l} \hat{T}(\pi[r])\right] \mid \boldsymbol{Z}[r]\right\}
\end{gathered}
$$

Substituting (57) yields:

$$
\begin{array}{r}
\Delta(\boldsymbol{Z}[r])+V \mathbb{E}\left\{\hat{y}_{0}(\pi[r]) \mid \boldsymbol{Z}[r]\right\} \leq B+C \\
+\mathbb{E}\left\{V \hat{y}_{0}\left(\pi^{*}[r]\right)+\sum_{l=1}^{L} Z_{l}[r]\left[\hat{y}_{l}\left(\pi^{*}[r]\right)-c_{l} \hat{T}\left(\pi^{*}[r]\right)\right]\right\}
\end{array}
$$

where $\pi^{*}[r]$ is any i.i.d. algorithm. As in Lemma 1 it can be shown that if the problem (54)-(56) is feasible, then for any $\delta>0$ there is an i.i.d. algorithm $\pi^{*}[r]$ such that:

$$
\begin{aligned}
\mathbb{E}\left\{\hat{y}_{0}\left(\pi^{*}[r]\right)\right\} & \leq y_{0}^{o p t}+\delta \\
\mathbb{E}\left\{\hat{y}_{l}\left(\pi^{*}[r]\right)\right\} / \mathbb{E}\left\{\hat{T}\left(\pi^{*}[r]\right)\right\} & \leq c_{l}+\delta \forall l \in\{1, \ldots, L\}
\end{aligned}
$$

Plugging the above into the right-hand-side of $(60)$ yields:

$$
\begin{array}{r}
\Delta(\boldsymbol{Z}[r])+V \mathbb{E}\left\{\hat{y}_{0}(\pi[r]) \mid \boldsymbol{Z}[r]\right\} \leq B+C+V\left(y_{0}^{o p t}+\delta\right) \\
+\sum_{l=1}^{L} Z_{l}[r]\left[\left(c_{l}+\delta\right) \mathbb{E}\left\{\hat{T}\left(\pi^{*}[r]\right)\right\}-\mathbb{E}\left\{c_{l} \hat{T}\left(\pi^{*}[r]\right)\right\}\right]
\end{array}
$$

Taking $\delta \rightarrow 0$ yields:

$$
\Delta(\boldsymbol{Z}[r])+V \mathbb{E}\left\{\hat{y}_{0}(\pi[r]) \mid \boldsymbol{Z}[r]\right\} \leq B+C+V y_{0}^{o p t}
$$

From this we obtain:

$$
\Delta(\boldsymbol{Z}[r]) \leq B+C+V\left(y_{0}^{\text {opt }}-y_{0}^{\min }\right)
$$

Thus, the quadratic Lyapunov drift is less than or equal to a constant, from which we obtain the result of part (a) by the same argument as in the proof of Theorem 1

To prove part (b), taking expectations of 61 yields:

$\mathbb{E}\{L(\boldsymbol{Z}[r+1])\}-\mathbb{E}\{L(\boldsymbol{Z}[r])\}+V \mathbb{E}\left\{y_{0}[r]\right\} \leq B+C+V y_{0}^{\text {opt }}$

Fix an integer $R>0$. Summing the above over $r \in\{0, \ldots R-$ 1 \} and dividing by $R$ gives:

$$
\frac{\mathbb{E}\{L(\boldsymbol{Z}[R])\}-\mathbb{E}\{L(\boldsymbol{Z}[0])\}}{R}+V \bar{y}_{0}[R] \leq B+C+V y_{0}^{\text {opt }}
$$

Rearranging terms and noting that $\mathbb{E}\{L(\boldsymbol{Z}[R])\} \geq 0$ proves the result.

\section{ApPendix D - Analysis of the Alternative Algorithm With Time Averaging}

Here we consider the original problem of minimizing $\bar{y}_{0} / \bar{T}$ subject to $\bar{y}_{l} / \bar{T} \leq c_{l}$ for $l \in\{1, \ldots, L\}$, and analyze the alternative algorithm (with time averaging) described in Section VI-B, Recall that queues $\boldsymbol{Z}[r]$ still operate according to [17). Define $\theta[0] \triangleq 0$, and for integers $r>0$ define $y_{0}^{a v}[r]$, $T^{a v}[r], \theta[r]$ by:

$y_{0}^{a v}[r] \triangleq \frac{1}{r} \sum_{i=0}^{r-1} y_{0}[r], T^{a v}[r] \triangleq \frac{1}{r} \sum_{i=0}^{r-1} T[r], \theta[r] \triangleq \frac{y_{0}^{a v}[r]}{T^{a v}[r]}$

Assume that we use a $C$-additive approximation for the policy selection step in (52), so that every frame $r$ we observe $\boldsymbol{Z}[r]$ and $\theta[r]$ and choose $\pi[r] \in \mathcal{P}$ to yield:

$$
\begin{array}{r}
\mathbb{E}\left\{V\left[\hat{y}_{0}(\pi[r])-\theta[r] \hat{T}(\pi[r])\right] \mid \boldsymbol{Z}[r], \theta[r]\right\} \\
+\mathbb{E}\left\{\sum_{l=1}^{L} Z_{l}[r]\left[\hat{y}_{l}(\pi[r])-c_{l} \hat{T}(\pi[r])\right] \mid \boldsymbol{Z}[r], \theta[r]\right\} \leq \\
C+V \mathbb{E}\left\{\hat{y}_{0}\left(\pi^{*}[r]\right)\right\}-\theta[r] \mathbb{E}\left\{\hat{T}\left(\pi^{*}[r]\right)\right\} \\
+\sum_{l=1}^{L} Z_{l}[r]\left[\mathbb{E}\left\{\hat{y}_{l}\left(\pi^{*}[r]\right)\right\}-c_{l} \mathbb{E}\left\{\hat{T}\left(\pi^{*}[r]\right)\right\}\right]
\end{array}
$$

We now make the following convergence assumption:

Assumption 1: There are constants $y_{0}^{a v}, T^{a v}$, and $\theta^{*} \triangleq y_{0}^{a v} / T^{a v}$, such that under the implementation we have the following convergence properties:

$$
\begin{array}{r}
\lim _{r \rightarrow \infty}\left(y_{0}^{a v}[r], T^{a v}[r], \theta[r]\right)=\left(y_{0}^{a v}, T^{a v}, \theta^{*}\right)(w \cdot p .1) \\
\lim _{r \rightarrow \infty}\left(\mathbb{E}\left\{y_{0}^{a v}[r]\right\}, \mathbb{E}\left\{T^{a v}[r]\right\}, \mathbb{E}\{\theta[r]\}\right)=\left(y_{0}^{a v}, T^{a v}, \theta^{*}\right)
\end{array}
$$

The equality (64) typically holds whenever (63) holds. Indeed, taking an expectation of (63) and assuming we can pass the expectation through the limit yields (64). We can exchange the limit and the expectation whenever $y_{0}^{a v}[r]$, $T^{a v}[r], \theta[r]$ are deterministically bounded for all $r$ and all 
sample paths, or when milder conditions hold that allow the Lebesgue dominated convergence theorem to be applied [24].

If (63) holds, it is easy to show that, with probability 1:

$$
\lim _{R \rightarrow \infty} \frac{1}{R} \sum_{r=0}^{R-1} \theta[r] T[r]=\theta^{*} T^{a v}=y_{0}^{a v}
$$

It is natural (and useful) to assume the above also holds in expectation:

Assumption 2: With $\theta^{*}, T^{a v}, y_{0}^{a v}$ as defined in Assumption 1, we have:

$$
\lim _{R \rightarrow \infty} \frac{1}{R} \sum_{r=0}^{R-1} \mathbb{E}\{\theta[r] T[r]\}=\theta^{*} T^{a v}=y_{0}^{a v}
$$

Theorem 4: Assume the constraints of the problem (12)(14) are feasible, that $\mathbb{E}\{L(\boldsymbol{Z}[0])\}<\infty$, and that we use a $C$-additive approximation as described above every frame. Then:

a) For all $l \in\{1, \ldots, L\}$ we have:

$$
\begin{aligned}
& \limsup _{R \rightarrow \infty} \bar{y}_{l}[R] / \bar{T}[R] \leq c_{l} \forall l \in\{1, \ldots, L\} \\
& \limsup _{R \rightarrow \infty} \frac{\sum_{r=0}^{R-1} y_{l}[r]}{\sum_{r=0}^{R-1} T[r]} \leq c_{l} \quad \text { (w.p.1) }
\end{aligned}
$$

b) If Assumptions 1 and 2 hold, then the achieved value of $\bar{y}_{0} / \bar{T}$ satisfies the following with probability 1 :

$$
\lim _{R \rightarrow \infty} y_{0}^{a v}[R] / T^{a v}[R] \leq \text { ratio }^{o p t}+(B+C) / V T^{\text {min }}
$$

where $B$ is defined as in (19) (with a conditional expectation that is also given $\theta[r]$ ).

Proof: Define $\Delta(\boldsymbol{Z}[r], \theta[r])$ as the conditional drift, conditioned also on knowledge of $\theta[r]$. This has the same form as (18), and so from (18) we have:

$$
\begin{array}{r}
\Delta(\boldsymbol{Z}[r], \theta[r])+V \mathbb{E}\left\{\hat{y}_{0}(\pi[r])-\theta[r] \hat{T}(\pi[r]) \mid \boldsymbol{Z}[r], \theta[r]\right\} \leq \\
B+V \mathbb{E}\left\{\hat{y}_{0}(\pi[r])-\theta[r] \hat{T}(\pi[r]) \mid \boldsymbol{Z}[r], \theta[r]\right\} \\
+\mathbb{E}\left\{\sum_{l=1}^{L} Z_{l}[r]\left[\hat{y}_{l}(\pi[r])-c_{l} \hat{T}(\pi[r])\right] \mid \boldsymbol{Z}[r], \theta[r]\right\}
\end{array}
$$

Using (62) in the right-hand-side above yields:

$$
\begin{aligned}
\Delta(\boldsymbol{Z}[r], \theta[r]) & +V \mathbb{E}\left\{\hat{y}_{0}(\pi[r])-\theta[r] \hat{T}(\pi[r]) \mid \boldsymbol{Z}[r], \theta[r]\right\} \leq \\
B & +C+V \mathbb{E}\left\{\hat{y}_{0}\left(\pi^{*}[r]\right)\right\}-\theta[r] \mathbb{E}\left\{\hat{T}\left(\pi^{*}[r]\right)\right\} \\
& +\sum_{l=1}^{L} Z_{l}[r]\left[\mathbb{E}\left\{\hat{y}_{l}\left(\pi^{*}[r]\right)\right\}-c_{l} \mathbb{E}\left\{\hat{T}\left(\pi^{*}[r]\right)\right\}\right]
\end{aligned}
$$

where $\pi^{*}[r]$ is any i.i.d. algorithm. Now plug the i.i.d. algorithm $\pi^{*}[r]$ from (15)-(16) into the right-hand-side of the above and take $\delta \rightarrow 0$ to yield:

$$
\begin{array}{r}
\Delta(\boldsymbol{Z}[r], \theta[r])+V \mathbb{E}\left\{\hat{y}_{0}(\pi[r])-\theta[r] \hat{T}(\pi[r]) \mid \boldsymbol{Z}[r], \theta[r]\right\} \leq \\
B+C+\text { Vratio }^{\text {opt }} \mathbb{E}\left\{\hat{T}\left(\pi^{*}[r]\right)\right\}-V \theta[r] \mathbb{E}\left\{\hat{T}\left(\pi^{*}[r]\right)\right\}
\end{array}
$$

The above has the form:

$$
\Delta(\boldsymbol{Z}[r], \theta[r]) \leq F
$$

for some finite constant $F$, and so part (a) holds by arguments similar to those in the proof of Theorem 1

Now taking expectations of 65 yields:

$$
\begin{array}{r}
\mathbb{E}\{L(\boldsymbol{Z}[r+1])\}-\mathbb{E}\{L(\boldsymbol{Z}[r])\} \\
+V\left[\mathbb{E}\left\{\hat{y}_{0}[r]\right\}-\mathbb{E}\{\theta[r] T[r]\}\right] \leq \\
B+C+\text { Vratio }^{\text {opt }} \mathbb{E}\left\{\hat{T}\left(\pi^{*}[r]\right)\right\} \\
-V \mathbb{E}\{\theta[r]\} \mathbb{E}\left\{\hat{T}\left(\pi^{*}[r]\right)\right\}
\end{array}
$$

Summing the above over $r \in\{0, \ldots, R-1\}$ and dividing by $R$ yields:

$$
\begin{array}{r}
\frac{\mathbb{E}\{L(\boldsymbol{Z}[R])\}-\mathbb{E}\{L(\boldsymbol{Z}[0])\}}{R} \\
+V\left[\bar{y}_{0}[R]-\frac{1}{R} \sum_{r=0}^{R-1} \mathbb{E}\{\theta[r] T[r]\}\right] \leq \\
B+C+\text { Vratio }^{\text {opt }} \mathbb{E}\left\{\hat{T}\left(\pi^{*}[r]\right)\right\} \\
-V \mathbb{E}\left\{\hat{T}\left(\pi^{*}[r]\right)\right\} \frac{1}{R} \sum_{r=0}^{R-1} \mathbb{E}\{\theta[r]\}
\end{array}
$$

Thus:

$$
\begin{array}{r}
V\left[\bar{y}_{0}[R]-\frac{1}{R} \sum_{r=0}^{R-1} \mathbb{E}\{\theta[r] T[r]\}\right] \leq \\
B+C+\text { Vratio }^{\text {opt }} \mathbb{E}\left\{\hat{T}\left(\pi^{*}[r]\right)\right\} \\
-V \mathbb{E}\left\{\hat{T}\left(\pi^{*}[r]\right)\right\} \frac{1}{R} \sum_{r=0}^{R-1} \mathbb{E}\{\theta[r]\}+\frac{\mathbb{E}\{L(\boldsymbol{Z}[0])\}}{R}
\end{array}
$$

However, by Assumptions 1 and 2:

$$
\lim _{R \rightarrow \infty}\left[\bar{y}_{0}[R]-\frac{1}{R} \sum_{r=0}^{R-1} \mathbb{E}\{\theta[r] T[r]\}\right]=y_{0}^{a v}-y_{0}^{a v}=0
$$

Thus, taking limits of the above yields:

$$
0 \leq B+C+\text { Vratio }^{o p t} T^{*}-V T^{*} \theta^{*}
$$

where we have defined $T^{*} \triangleq \mathbb{E}\left\{\hat{T}\left(\pi^{*}[r]\right)\right\}$, and we have used the fact that if $\mathbb{E}\{\theta[r]\} \rightarrow \theta^{*}$, then so does its time average. Rearranging terms yields:

$$
y_{0}^{a v} / T^{a v} \triangleq \theta^{*} \leq \text { ratio }^{o p t}+\frac{B+C}{V T^{*}} \leq \text { ratio }^{o p t}+\frac{B+C}{V T^{m i n}}
$$

This proves Theorem 4

\section{APPENDiX E - VARIATION ON JENSEN's INEQUALITy}

Here we prove part (a) of Lemma 3. Recall that $(T, \gamma)$ is a random vector with arbitrary joint distribution such that $T>0$ and $\gamma \in \mathcal{R}$ with probability 1 , and $0<\mathbb{E}\{T\}<$ $\infty$. Define $\{(T[r], \gamma[r])\}_{r=0}^{\infty}$ as an infinite sequence of i.i.d. random vectors, each distributed the same as $(T, \gamma)$. Define ${ }^{(65)} t[0]=0$, and for integers $r>0$ define $t[r] \triangleq \sum_{i=0}^{r-1} T[i]$. The value of $t[r]$ can be viewed as the $r$ th renewal time in a process with i.i.d. renewal durations of size $T[r]$. Define $\gamma(t)$ as a random vector process defined over continuous time $t \geq 0$, 
taking the value $\gamma[r]$ whenever $t$ is in the $r$ th renewal interval, so that:

$$
\gamma(t)=\gamma[r] \text { if and only if } t[r] \leq t<t[r+1]
$$

Therefore, for any integer $r>0$ :

$$
\frac{1}{t[r]} \int_{0}^{t[r]} \phi(\gamma(t)) d t=\frac{\sum_{i=0}^{r-1} T[i] \phi(\gamma[i])}{\sum_{i=0}^{r-1} T[i]}
$$

On the other hand, by Jensen's inequality for integrals of concave functions, we have:

$$
\begin{aligned}
\frac{1}{t[r]} \int_{0}^{t[r]} \phi(\gamma(t)) d t & \leq \phi\left(\frac{1}{t[r]} \int_{0}^{t[r]} \gamma(t) d t\right) \\
& =\phi\left(\frac{\sum_{i=0}^{r-1} \gamma[i] T[i]}{\sum_{i=0}^{r-1} T[i]}\right)
\end{aligned}
$$

Thus, for all integers $r>0$ we have:

$$
\frac{\frac{1}{r} \sum_{i=0}^{r-1} T[i] \phi(\gamma[i])}{\frac{1}{r} \sum_{i=0}^{r-1} T[i]} \leq \phi\left(\frac{\frac{1}{r} \sum_{i=0}^{r-1} \gamma[i] T[i]}{\frac{1}{r} \sum_{i=0}^{r-1} T[i]}\right)
$$

However, by the law of large numbers, we know:

$$
\begin{aligned}
\lim _{r \rightarrow \infty} \frac{1}{r} \sum_{i=0}^{r-1} T[i] & =\mathbb{E}\{T\} \quad(w \cdot p .1) \\
\lim _{r \rightarrow \infty} \frac{1}{r} \sum_{i=0}^{r-1} T[i] \phi(\gamma[i]) & =\mathbb{E}\{T \phi(\gamma)\} \quad \text { (w.p. } 1) \\
\lim _{r \rightarrow \infty} \frac{1}{r} \sum_{i=0}^{r-1} \gamma[i] T[i] & =\mathbb{E}\{T \gamma\} \quad \text { (w.p. } 1)
\end{aligned}
$$

Taking limits of the above as $r \rightarrow \infty$ in (66) and using the above identities together with continuity of $\phi(\gamma)$ proves that:

$$
\frac{\mathbb{E}\{T \phi(\gamma)\}}{\mathbb{E}\{T\}} \leq \phi\left(\frac{\mathbb{E}\{T \gamma\}}{\mathbb{E}\{T\}}\right)
$$

\section{APPENDIX F - ANALYSIS OF UTILITY OptimizATION}

Here we prove Theorem 2 For simplicity, assume that the set $\Gamma$ of all expectations $[\boldsymbol{y}[r], \boldsymbol{x}[r], T[r]]$ under i.i.d. algorithms is closed. Then, similar to Lemma 1, it can be shown that if the problem (42)-(44) is feasible, then there is an i.i.d. algorithm $\pi^{*}[r]$ and a vector $\gamma \in \mathcal{R}$ such that:

$$
\begin{aligned}
\phi\left(\gamma^{*}\right) & =\text { util } \text { opt }^{\text {t }} \\
\frac{\mathbb{E}\left\{\hat{x}_{m}\left(\pi^{*}[r]\right)\right\}}{\mathbb{E}\left\{\hat{T}\left(\pi^{*}[r]\right)\right\}} & =\gamma_{m}^{*} \forall m \in\{1, \ldots, M\} \\
\frac{\mathbb{E}\left\{\hat{y}_{l}\left(\pi^{*}[r]\right)\right\}}{\mathbb{E}\left\{\hat{T}\left(\pi^{*}[r]\right)\right\}} & \leq c_{l} \forall l \in\{1, \ldots, L\}
\end{aligned}
$$

If the set $\Gamma$ is not closed, then (67)- 69) can be modified to show they hold to within any $\delta>0$, and the same result we derive below can be recovered by taking $\delta \rightarrow 0$, as in the proof of Theorem 1

Now define $\boldsymbol{Q}[r] \triangleq[\boldsymbol{Z}[r] ; \boldsymbol{G}[r]]$ as the collection of all queues, and define the Lyapunov function:

$$
L(\boldsymbol{Q}[r]) \triangleq \frac{1}{2} \sum_{l=1}^{L} Z_{l}[r]^{2}+\frac{1}{2} \sum_{m=1}^{M} G_{m}[r]^{2}
$$

For simplicity, assume initial conditions satisfy $Z_{l}[0]=G_{m}[0]=0$ for all $l$ and $m$. Define $\Delta(\boldsymbol{Q}[r]) \triangleq \mathbb{E}\{L(\boldsymbol{Q}[r+1])-L(\boldsymbol{Q}[r]) \mid \boldsymbol{Q}[r]\}$. By an argument similar to that given in Lemma 2 , we can square the queue update equations (17) and (41) to obtain the following drift-plus-penalty bound:

$$
\begin{array}{r}
\Delta(\boldsymbol{Q}[r])-V \mathbb{E}\{T[r] \phi(\gamma[r]) \mid \boldsymbol{Q}[r]\} \leq \\
D-V \mathbb{E}\{T[r] \phi(\gamma[r]) \mid \boldsymbol{Q}[r]\} \\
+\mathbb{E}\left\{\sum_{l=1}^{L} Z_{l}[r]\left[y_{l}[r]-c_{l} T[r]\right] \mid \boldsymbol{Q}[r]\right\} \\
+\mathbb{E}\left\{\sum_{m=1}^{M} G_{m}[r]\left[T[r] \gamma_{m}[r]-x_{m}[r]\right] \mid \boldsymbol{Q}[r]\right\}
\end{array}
$$

where $D$ is defined in (45). This drift-plus-penalty bound can be rearranged as follows:

$$
\begin{array}{r}
\Delta(\boldsymbol{Q}[r])-V \mathbb{E}\{T[r] \phi(\gamma[r]) \mid \boldsymbol{Q}[r]\} \leq \\
D-\mathbb{E}\left\{\left[V \phi(\gamma[r])-\sum_{m=1}^{M} G_{m}[r] \gamma_{m}[r]\right] T[r] \mid \boldsymbol{Q}[r]\right\} \\
+\mathbb{E}\left\{\sum_{l=1}^{L} Z_{l}[r] y_{l}[r]-\sum_{m=1}^{M} G_{m}[r] x_{m}[r] \mid \boldsymbol{Q}[r]\right\} \\
-\sum_{l=1}^{L} Z_{l}[r] c_{l} \mathbb{E}\{T[r] \mid \boldsymbol{Q}[r]\}
\end{array}
$$

However, by the auxiliary variable update algorithm, we know that for every frame $r$ :

$$
V \phi(\gamma[r])-\sum_{m=1}^{M} G_{m}[r] \gamma_{m}[r] \geq V \phi\left(\gamma^{*}\right)-\sum_{m=1}^{M} G_{m}[r] \gamma_{m}^{*}
$$

for any vector $\gamma^{*}=\left(\gamma_{1}^{*}, \ldots, \gamma_{M}^{*}\right) \in \mathcal{R}$. Further, because we use a $C$-additive approximation when choosing $\pi[r] \in \mathcal{P}$, every frame $r$ we have:

$$
\begin{gathered}
\frac{\mathbb{E}\left\{\sum_{l=1}^{L} Z_{l}[r] \hat{y}_{l}(\pi[r])-\sum_{m=1}^{M} G_{m}[r] \hat{x}_{m}(\pi[r]) \mid \boldsymbol{Q}[r]\right\}}{\mathbb{E}\{\hat{T}(\pi[r]) \mid \boldsymbol{Q}[r]\}} \leq C \\
+\frac{\sum_{l=1}^{L} Z_{l}[r] \mathbb{E}\left\{\hat{y}_{l}\left(\pi^{*}[r]\right)\right\}-\sum_{m=1}^{M} G_{m}[r] \mathbb{E}\left\{\hat{x}_{m}\left(\pi^{*}[r]\right)\right\}}{\mathbb{E}\left\{\hat{T}\left(\pi^{*}[r]\right)\right\}}
\end{gathered}
$$

where $\pi^{*}[r]$ is any i.i.d. algorithm. Plugging the above two inequalities into the right-hand-side of (70) yields:

$$
\begin{aligned}
& \Delta(\boldsymbol{Q}[r])-V \mathbb{E}\{T[r] \phi(\gamma[r]) \mid \boldsymbol{Q}[r]\} \leq \\
& D-\left[V \phi\left(\boldsymbol{\gamma}^{*}\right)-\sum_{m=1}^{M} G_{m}[r] \gamma_{m}^{*}\right] \mathbb{E}\{T[r] \mid \boldsymbol{Q}[r]\} \\
& +C \mathbb{E}\{T[r] \mid \boldsymbol{Q}[r]\}+\mathbb{E}\{T[r] \mid \boldsymbol{Q}[r]\} \times \\
& {\left[\sum_{l=1}^{L} Z_{l}[r] \frac{\mathbb{E}\left\{\hat{y}_{l}\left(\pi^{*}[r]\right)\right\}}{\mathbb{E}\left\{\hat{T}\left(\pi^{*}[r]\right)\right\}}-\sum_{m=1}^{M} G_{m}[r] \frac{\mathbb{E}\left\{\hat{x}_{m}\left(\pi^{*}[r]\right)\right\}}{\mathbb{E}\left\{\hat{T}\left(\pi^{*}[r]\right)\right\}}\right]} \\
& -\sum_{l=1}^{L} Z_{l}[r] c_{l} \mathbb{E}\{T[r] \mid \boldsymbol{Q}[r]\}
\end{aligned}
$$


where $\gamma^{*}$ is any vector in $\mathcal{R}$, and $\pi^{*}[r]$ is any i.i.d. algorithm Plugging the vector $\gamma^{*}$ and the i.i.d. algorithm $\pi^{*}[r]$ from (67)- 69) into the right-hand-side of (71) yields:

$$
\begin{array}{r}
\Delta(\boldsymbol{Q}[r])-V \mathbb{E}\{T[r] \phi(\gamma[r]) \mid \boldsymbol{Q}[r]\} \leq \\
D+C \mathbb{E}\{T[r] \mid \boldsymbol{Q}[r]\}-V \text { util }^{\text {opt }} \mathbb{E}\{T[r] \mid \boldsymbol{Q}[r]\}
\end{array}
$$

It follows from the above that $\Delta(\boldsymbol{\Theta}[r]) \leq F$ for some constant $F$. Thus, we know all queues $Z_{l}[r]$ and $G_{m}[r]$ are mean rate stable and rate stable [25]. This proves part (a) by an argument similar to that given in the proof of Theorem 1

To prove part (b), we have by taking expectations of (72):

$$
\begin{array}{r}
\mathbb{E}\{L(\boldsymbol{Q}[r+1])\}-\mathbb{E}\{L(\boldsymbol{Q}[r])\}-V \mathbb{E}\{T[r] \phi(\gamma[r])\} \leq \\
D+C \mathbb{E}\{T[r]\}-V u t i l^{\text {opt }} \mathbb{E}\{T[r]\}
\end{array}
$$

Summing the above over $r \in\{0, \ldots, R-1\}$, dividing by $R V$, recalling our assumption that $L(\boldsymbol{Q}[0])=0$, removing the nonnegative term $\mathbb{E}\{L(\boldsymbol{Q}[R])\}$, and rearranging terms yields:

$$
\frac{1}{R} \sum_{r=0}^{R-1} \mathbb{E}\{T[r] \phi(\gamma[r])\} \geq u t i l^{o p t} \bar{T}[r]-\frac{D+C \bar{T}[R]}{V}
$$

where we have used $\bar{T}[R] \triangleq \frac{1}{R} \sum_{r=0}^{R-1} \mathbb{E}\{T[r]\}$. Dividing both sides by $\bar{T}[R]$ and using the variation on Jensen's inequality (Lemma 3) yields:

$$
\phi\left(\frac{\frac{1}{R} \sum_{r=0}^{R-1} \mathbb{E}\{T[r] \gamma[r]\}}{\bar{T}[R]}\right) \geq u t i l^{\text {opt }}-\frac{D}{V \bar{T}[R]}-\frac{C}{V}
$$

The above holds for all integers $R>0$.

However, we have from (41) (similar to derivation of (34)):

$$
\frac{\mathbb{E}\{\boldsymbol{G}[R]\}}{R}+\overline{\boldsymbol{x}}[R] \geq \frac{1}{R} \sum_{r=0}^{R-1} \mathbb{E}\{T[r] \boldsymbol{\gamma}[r]\}
$$

where the above vector inequality is taken entrywise. Using this in (73) together with the fact that $\phi(\gamma)$ is entrywise nondecreasing, we have:

$$
\phi\left(\frac{\overline{\boldsymbol{x}}[R]+\mathbb{E}\{\boldsymbol{G}[R]\} / R}{\bar{T}[R]}\right) \geq u t i l^{o p t}-\frac{D}{V \bar{T}[R]}-\frac{C}{V}
$$

Using the fact that $\bar{T}[R] \geq T^{\text {min }}$ gives:

$$
\phi\left(\frac{\overline{\boldsymbol{x}}[R]+\mathbb{E}\{\boldsymbol{G}[R]\} / R}{\bar{T}[R]}\right) \geq u t i l^{\text {opt }}-\frac{D}{V T^{\min }}-\frac{C}{V}
$$

Now recall that all queues $G_{m}[r]$ are mean rate stable, so that:

$$
\lim _{R \rightarrow \infty} \frac{\mathbb{E}\{\boldsymbol{G}[R]\}}{R}=\mathbf{0}
$$

Taking a liminf of (74) as $R \rightarrow \infty$ and using continuity of $\phi(\cdot)$ proves part (b) of Theorem 2 .

\section{APPENDiX G - SiMUlation Details AND \\ DETERMINISTIC BOUNDS}

Here we provide simulation details for the particular system of Section VII We also show that deterministic bounds on the constraint violations are computable if the $I^{\max }$ parameter is chosen suitably large.

\section{A. Simulation Details}

The bisection algorithm for the above example was implemented by computing $\operatorname{val}(\theta)$ in 50 , where the term for each sample $\boldsymbol{\eta}_{w}$ is found by observing $\boldsymbol{Z}[r]$ and choosing $l[r] \in\{1, \ldots, 5\}$ and Idle $[r] \in\left[0, I^{\max }\right]$ to minimize the following deterministic expression:

$$
\begin{array}{r}
-\operatorname{Vqual}_{l[r]}[r]+\sum_{l=1}^{L} Z_{l}[r][ \\
{\left[0.5+P^{\operatorname{tran}} T_{l}^{\operatorname{tran}}[r] 1_{\{l[r]=l\}}\right]} \\
-\theta\left(0.5+T_{l[r]}^{\operatorname{tran}}[r]+I d l e[r]\right)
\end{array}
$$

This is solved by choosing Idle $[r]=0$ whenever $\theta \leq 0$, and Idle $[r]=I^{\max }$ if $\theta>0$, and choosing $l[r]$ as the index $l \in\{1, \ldots, 5\}$ that minimizes:

$$
-\operatorname{Vqual}_{l}[r]+\left(Z_{l}[r] P^{\text {tran }}-\theta\right) T_{l}^{\text {tran }}[r]
$$

The alternative algorithm with time averaging is implemented by observing $\boldsymbol{Z}[r]$ and $\theta[r]$ and minimizing (52), which in this context amounts to choosing $l[r] \in\{1, \ldots, 5\}$, Idle $[r] \in\left[0, I^{\max }\right]$ to minimize:

$$
-\operatorname{Vqual}_{l[r]}[r]-V \theta[r]\left(T_{l[r]}^{\operatorname{tran}}[r]+I d l e[r]\right)+
$$

$\sum_{l=1}^{L} Z_{l}[r]\left[P^{\text {tran }} T_{l}^{\text {tran }}[r] 1_{\{l[r]=l\}}-c_{l}\left(T_{l[r]}^{\text {tran }}[r]+\right.\right.$ Idle $\left.\left.[r]\right)\right]$

This amounts to choosing Idle $[r]=0$ whenever $V \theta[r]+$ $\sum_{l=1}^{L} Z_{l}[r] c_{l} \leq 0$, and Idle $[r]=I^{\max }$ else, and choosing $l[r]$ as the index $l \in\{1, \ldots, 5\}$ that minimizes:

$-\operatorname{Vqual}_{l}[r]-T_{l}^{\text {tran }}[r]\left[V \theta[r]-Z_{l}[r] P^{\text {tran }}+\sum_{k=1}^{L} Z_{k}[r] c_{k}\right]$

\section{B. Deterministic Queue Bounds}

Here we show that, if $I^{\max }$ is chosen to be suitably large, then the drift-plus-penalty ratio algorithm for this context yields deterministic bounds on $Z_{l}[r]$. The ratio to minimize is:

$$
\frac{\mathbb{E}\left\{- \text { Vqual }_{l[r]}[r]+\sum_{l=1}^{L} Z_{l}[r] \hat{y}_{l}(\pi[r]) \mid \boldsymbol{Z}[r]\right\}}{\mathbb{E}\left\{0.5+T_{l[r]}^{\text {tran }}[r]+\operatorname{Idle}[r] \mid \boldsymbol{Z}[r]\right\}}
$$

Because $\hat{y}_{l}(\pi[r]) \geq 0.5$ for all $l$ and all policy choices, and - Vqual $_{l[r]}[r] \geq-5 V$ for all policy choices, the numerator above is positive whenever:

$$
\sum_{l=1}^{L} Z_{l}[r](0.5)>-5 V
$$

In particular, the algorithm chooses $I d l e[r]=I^{\max }$ whenever $Z_{l}[r]>10 \mathrm{~V}$ for any queue $l \in\{1, \ldots, L\}$.

Recall that the $Z_{l}[r]$ update is given by:

$$
Z_{l}[r+1]=\max \left[Z_{l}[r]+\hat{y}_{l}(\pi[r])-0.25 \hat{T}(\pi[r]), 0\right]
$$

Because $\hat{y}_{l}(\pi[r]) \leq 0.5+2.5 P^{\text {tran }}$, and $\hat{T}(\pi[r]) \geq 1.0+$ Idle $[r]$, whenever Idle $[r]=I^{\max }$ we have:

$Z_{l}[r+1] \leq \max \left[Z_{l}[r]+0.5+2.5 P^{\operatorname{tran}}-0.25\left(1.0+I^{\max }\right), 0\right]$ 
Therefore, $Z_{l}[r]$ cannot increase on the next slot if $I d l e[r]=$ $I^{\max }$ and if:

$$
I^{\max } \geq \frac{0.5+2.5 P^{\text {tran }}-0.25}{0.25}=1.0+10 P^{\text {tran }}
$$

This means that if any queue $Z_{l}[r]$ exceeds $10 \mathrm{~V}$, then Idle $[r]=I^{\max }$ and it cannot increase further. Because the maximum increase in $Z_{l}[r]$ is $0.5+2.5 P^{\text {tran }}-0.25$, for all $l \in\{1, \ldots, 5\}$ and all frames $r$ we have:

$$
0 \leq Z_{l}[r] \leq 10 V+0.5+2.5 P^{\text {tran }}-0.25=d_{1} V+d_{2}
$$

provided that this inequality holds for $r=0$, where $d_{1} \triangleq 10$ and $d_{2} \triangleq 0.25+2.5 P^{\text {tran }}$. Thus, from (33) we have for all $l \in\{1, \ldots, L\}$ and all integers $R>0$ :

$$
\begin{aligned}
\frac{\sum_{r=0}^{R-1} y_{l}[r]}{\sum_{r=0}^{R-1} T[r]} & \leq c_{l}+\frac{d_{1} V+d_{2}}{\sum_{r=0}^{R-1} T[r]} \\
& \leq c_{l}+\frac{d_{1} V+d_{2}}{R}
\end{aligned}
$$

where we have used the fact that $T[r] \geq 1.0$ for all $r$. This provides a deterministic guarantee on the worst-case constraint violation over any interval of frames starting at frame 0 . The deviation is $O(1 / R)$, which decays at a faster rate than the general $O(\sqrt{1 / R})$ bound in (25).

\section{ApPendix H - Convergence Under A Slater CONDITION}

We first present a definition and two theorems from [25]. Define $\mathcal{H}[0] \triangleq \boldsymbol{Z}[0]$, and for integers $r>0$ define $\mathcal{H}[r]$ as the system history up to frame $r$, being the queue values up to and including frame $r$, and the penalties up to but not including frame $r$ :

$$
\mathcal{H}[r] \triangleq[\boldsymbol{Z}[0], \boldsymbol{Z}[1], \ldots, \boldsymbol{Z}[r], \boldsymbol{y}[0], \boldsymbol{y}[1], \ldots, \boldsymbol{y}[r-1]]
$$

Define $L(\boldsymbol{Z}[r])$ by:

$$
L(\boldsymbol{Z}[r]) \triangleq \frac{1}{2} \sum_{l=1}^{L} Z_{l}[r]^{2}
$$

Define $\Delta(\mathcal{H}[r])$ by:

$$
\Delta(\mathcal{H}[r]) \triangleq \mathbb{E}\{L(\boldsymbol{Z}[r+1])-L(\boldsymbol{Z}[r]) \mid \mathcal{H}[r]\}
$$

Suppose that per-frame changes in the $Z_{l}[r]$ queues have bounded conditional fourth moments, regardless of past history, so that there is a constant $D>0$ such that for all $l \in\{1, \ldots, L\}$, all frames $r$, and all possible $\mathcal{H}[r]$ we have:

$$
\mathbb{E}\left\{\left(Z_{l}[r+1]-Z_{l}[r]\right)^{4} \mid \mathcal{H}[r]\right\} \leq C
$$

Theorem 5: (from [25]) Suppose $L(\boldsymbol{Z}[r])$ is the quadratic Lyapunov function in (76), and that per-frame changes in the queues $Z_{[} r$ h have conditional bounded fourth moments, so that (77) holds. Suppose that $\mathbb{E}\{L(\boldsymbol{Z}[0])\}<\infty$, and that there are constants $B>0, \epsilon>0$ such that for all $r$ and all possible $\mathcal{H}[r]$, we have:

$$
\Delta(\mathcal{H}[r]) \leq B-\epsilon \sum_{l=1}^{L} Z_{l}[r]
$$

Then for all $l \in\{1, \ldots, L\}$ we have:

$\sum_{r=1}^{\infty} \frac{\mathbb{E}\left\{Z_{l}[r]^{2}\right\}}{r^{2}}<\infty, \limsup _{R \rightarrow \infty} \frac{1}{R} \sum_{r=0}^{R-1} Z_{l}[r] \leq B / \epsilon \quad$ (w.p.1)
Theorem 6: (from [25]) Suppose $L(\boldsymbol{Z}[r])$ is the quadratic Lyapunov function in (76), and that per-frame changes in the queues $Z_{l}[r]$ have conditional bounded fourth moments, so that (77) holds. Suppose that $\beta[r]$ is some additional process related to the system, and that $\beta[0]$ and $L(\boldsymbol{Z}[0])$ are finite with probability 1. Suppose that:

$$
\sum_{r=1}^{\infty} \frac{\mathbb{E}\left\{\beta[r]^{2}+Z_{l}[r]^{2}\right\}}{r^{2}}<\infty
$$

If there are constants $V>0, B>0, \beta^{*}$ such that for all frames $r$ and all possible $\mathcal{H}[r]$ we have:

$$
\Delta(\mathcal{H}[r])+V \mathbb{E}\{\beta[r] \mid \mathcal{H}[r]\} \leq B+V \beta^{*}
$$

Then:

$$
\limsup _{R \rightarrow \infty} \frac{1}{R} \sum_{r=0}^{R-1} \beta[r] \leq \beta^{*}+B / V \quad(w \cdot p .1)
$$

We now prove the following result.

Theorem 7: Suppose the same assumptions as in Theorem 1 hold, and for simplicity assume that $C=0$. Additionally assume the fourth moment boundedness condition (77) holds, that second moments of $y_{0}[r]$ are bounded by the same constant for all $r$, and that there exists an $\epsilon>0$ and an i.i.d. algorithm $\pi^{*}[r]$ such that:

$$
\frac{\mathbb{E}\left\{\hat{y}_{l}\left(\pi^{*}[r]\right)\right\}}{\mathbb{E}\left\{\hat{T}\left(\pi^{*}[r]\right)\right\}} \leq c_{l}-\epsilon \forall l \in\{1, \ldots, L\}
$$

Then:

(a) We have:

$$
\limsup _{R \rightarrow \infty} \frac{1}{R} \sum_{r=0}^{R-1}\left[y_{0}[r]-T[r] \text { ratio }^{o p t}\right] \leq \frac{B}{V}(w . p .1)
$$

(b) We have:

$$
\limsup _{R \rightarrow \infty} \frac{\sum_{r=0}^{R-1} y_{0}[r]}{\sum_{r=0}^{R-1} T[r]} \leq \text { ratio }^{\text {opt }}+\frac{B}{V T^{\min }} \text { (w.p.1) }
$$

Proof: (Theorem 7 part (a)) Use of the history-based drift $\Delta(\mathcal{H}[r])$ is required for the above theorems. However, manipulations with this drift are almost the same, and the same proof of Theorem 1 can be repeated to line (27) to show that if the conditions of the theorem hold, then (compare with 27) and note that $C=0$ ):

$$
\begin{gathered}
\Delta(\mathcal{H}[r])+V \mathbb{E}\left\{\hat{y}_{0}(\pi[r]) \mid \mathcal{H}[r]\right\} \leq \\
B+\mathbb{E}\{\hat{T}(\pi[r]) \mid \mathcal{H}[r]\} \text { Vratio }^{\text {opt }}
\end{gathered}
$$

Rearranging terms yields:

$\Delta(\mathcal{H}[r])+V \mathbb{E}\left\{\hat{y}_{0}(\pi[r])-\hat{T}(\pi[r])\right.$ ratio $\left.^{\text {opt }} \mid \mathcal{H}[r]\right\} \leq B(80)$

This is the same as condition (78) with $\beta[r] \triangleq \hat{y}_{0}(\pi[r])-$ $\hat{T}(\pi[r])$ ratio $^{o p t}$ and $\beta^{*} \triangleq 0$. By Theorem 6 , it follows that if the fourth moment boundedness condition (77) holds, and if:

$$
\sum_{r=1}^{\infty} \frac{\mathbb{E}\left\{\beta[r]^{2}+Z_{l}[r]^{2}\right\}}{r^{2}}<\infty
$$

then we can conclude the result of part (a). 
It suffices to show that 81 holds. However, since we know that second moments of $y_{0}[r]$ and $T[r]$ are bounded by the same finite constant for all $r$, it is easy to see that:

$$
\sum_{r=1}^{\infty} \frac{\mathbb{E}\left\{\beta[r]^{2}\right\}}{r^{2}}<\infty
$$

It suffices to show that $\sum_{r=1}^{\infty} Z_{l}[r]^{2} / r^{2}<\infty$. To this end, the proof of Theorem 1 can be repeated to line (26) to show that (compare with (26)):

$$
\begin{gathered}
\Delta(\mathcal{H}[r])+V \mathbb{E}\left\{\hat{y}_{0}(\pi[r]) \mid \mathcal{H}[r]\right\} \leq B+ \\
\mathbb{E}\{\hat{T}(\pi[r]) \mid \mathcal{H}[r]\}\left[C+\frac{\mathbb{E}\left\{V \hat{y}_{0}\left(\pi^{*}[r]\right)+\sum_{l=1}^{L} Z_{l}[r] \hat{y}_{l}\left(\pi^{*}[r]\right)\right\}}{\mathbb{E}\left\{\hat{T}\left(\pi^{*}[r]\right)\right\}}\right] \\
-\sum_{l=1}^{L} Z_{l}[r] c_{l} \mathbb{E}\{\hat{T}(\pi[r]) \mid \mathcal{H}[r]\}
\end{gathered}
$$

where $\pi^{*}[r]$ is from any i.i.d. algorithm. Plugging (79) into the right-hand-side of (82) yields:

$$
\begin{array}{r}
\Delta(\mathcal{H}[r])+V \mathbb{E}\left\{\hat{y}_{0}(\pi[r]) \mid \mathcal{H}[r]\right\} \leq \\
B+\mathbb{E}\{\hat{T}(\pi[r]) \mid \mathcal{H}[r]\}\left[C+\frac{\mathbb{E}\left\{V \hat{y}_{0}\left(\pi^{*}[r]\right)\right\}}{\left.\mathbb{E}\left\{\hat{T}\left(\pi^{*}[r]\right)\right\}\right]}\right. \\
-\mathbb{E}\{\hat{T}(\pi[r]) \mid \mathcal{H}[r]\} \sum_{l=1}^{L} Z_{l}[r] \epsilon
\end{array}
$$

In particular, because all conditional expectations are assumed to be upper and lower bounded (and because $T^{\text {min }}>0$ ), we have:

$$
\Delta(\mathcal{H}[r]) \leq B_{1}-\epsilon T^{\text {min }} \sum_{l=1}^{L} Z_{l}[r]
$$

where $B_{1}$ is a positive constant. It follows by Theorem 5 that $\sum_{r=1}^{\infty} \mathbb{E}\left\{Z_{l}[r]^{2}\right\} / r^{2}<\infty$ for all $l \in\{1, \ldots, L\}$.

Proof: (Theorem 7 part (b)) First note that if the limsup of a function $f[r]$ is upper bounded by a positive constant, then the limsup of $[f[r]]^{+}$is bounded by the same constant, where $[f[r]]^{+} \triangleq \max [f[r], 0]$. Thus, by part (a) we have:

$$
\limsup _{R \rightarrow \infty}\left[\frac{1}{R} \sum_{r=0}^{R-1}\left[y_{0}[r]-T[r] \text { ratio }^{\text {opt }}\right]\right]^{+} \leq \frac{B}{V} \text { (w.p.1) }
$$

Next note that because $\mathbb{E}\{T[r] \mid \mathcal{H}[r]\} \geq T^{\text {min }}>0$ for all $r$ and all $\mathcal{H}[r]$, and second moments of $T[r]$ are bounded by the same finite constant for all $r$, it can be shown that [25]:

$$
\limsup _{R \rightarrow \infty}\left[\frac{R}{\sum_{r=0}^{R-1} T[r]}\right] \leq 1 / T^{\text {min }} \text { (w.p.1) }
$$

We then have:

$$
\begin{array}{r}
{\left[\frac{\sum_{r=0}^{R-1} y_{0}[r]}{\sum_{r=0}^{R-1} T[r]}-\text { ratio }^{o p t}\right] \leq\left[\frac{R}{\sum_{r=0}^{R-1} T[r]}\right] \times} \\
{\left[\frac{1}{R} \sum_{r=0}^{R-1}\left[y_{0}[r]-T[r] \text { ratio }^{\text {opt }}\right]\right]^{+}}
\end{array}
$$

Taking limsups of the above and using the fact that the limsup of a product of non-negative functions is less than or equal to the product of the lim sups yields:

$$
\limsup _{R \rightarrow \infty}\left[\frac{\sum_{r=0}^{R-1} y_{0}[r]}{\sum_{r=0}^{R-1} T[r]}-\text { ratio }^{\text {opt }}\right] \leq \frac{1}{T^{\min }} \frac{B}{V}(w \cdot p .1)
$$

\section{REFERENCES}

[1] M. J. Neely. Dynamic optimization and learning for renewal systems Proc. Asilomar Conf. on Signals, Systems, and Computers, Nov. 2010.

[2] R. Gallager. Discrete Stochastic Processes. Kluwer Academic Publishers, Boston, 1996

[3] S. Ross. Introduction to Probability Models. Academic Press, 8th edition, Dec. 2002.

[4] L. Georgiadis, M. J. Neely, and L. Tassiulas. Resource allocation and cross-layer control in wireless networks. Foundations and Trends in Networking, vol. 1, no. 1, pp. 1-149, 2006.

[5] M. J. Neely. Stochastic optimization for markov modulated networks with application to delay constrained wireless scheduling. Proc. IEEE Conf. on Decision and Control (CDC), Shanghai, China, Dec. 2009.

[6] F. J. Vázquez Abad and V. Krishnamurthy. Policy gradient stochastic approximation algorithms for adaptive control of constrained time varying markov decision processes. Proc. IEEE Conf. on Decision and Control, Dec. 2003.

[7] D. V. Djonin and V. Krishnamurthy. q-learning algorithms for constrained markov decision processes with randomized monotone policies: Application to mimo transmission control. IEEE Transactions on Signal Processing, vol. 55, no. 5, pp. 2170-2181, May 2007.

[8] N. Salodkar, A. Bhorkar, A. Karandikar, and V. S. Borkar. An on-line learning algorithm for energy efficient delay constrained scheduling over a fading channel. IEEE Journal on Selected Areas in Communications, vol. 26, no. 4, pp. 732-742, May 2008.

[9] F. Fu and M. van der Schaar. A systematic framework for dynamically optimizing multi-user video transmission. IEEE Journal on Selected Areas in Communications, vol. 28, no. 3, pp. 308-320, April 2010.

[10] F. Fu and M. van der Schaar. Decomposition principles and online learning in cross-layer optimization for delay-sensitive applications. IEEE Trans. Signal Processing, vol. 58, no. 3, pp. 1401-1415, March 2010.

[11] M. J. Neely. Dynamic Power Allocation and Routing for Satellite and Wireless Networks with Time Varying Channels. $\mathrm{PhD}$ thesis, Massachusetts Institute of Technology, LIDS, 2003.

[12] M. J. Neely. Energy optimal control for time varying wireless networks. IEEE Transactions on Information Theory, vol. 52, no. 7, pp. 2915-2934, July 2006.

[13] M. J. Neely, E. Modiano, and C. Li. Fairness and optimal stochastic control for heterogeneous networks. Proc. IEEE INFOCOM, March 2005.

[14] M. J. Neely. Stochastic Network Optimization with Application to Communication and Queueing Systems. Morgan \& Claypool, 2010.

[15] A. Eryilmaz and R. Srikant. Fair resource allocation in wireless networks using queue-length-based scheduling and congestion control. IEEE/ACM Transactions on Networking, vol. 15, no. 6, pp. 1333-1344, Dec. 2007.

[16] A. Stolyar. Maximizing queueing network utility subject to stability: Greedy primal-dual algorithm. Queueing Systems, vol. 50, no. 4, pp. 401-457, 2005.

[17] A. Stolyar. Greedy primal-dual algorithm for dynamic resource allocation in complex networks. Queueing Systems, vol. 54, no. 3, pp. 203-220, 2006.

[18] Q. Li and R. Negi. Scheduling in wireless networks under uncertainties: A greedy primal-dual approach. Arxiv Technical Report: arXiv:1001:2050v2, June 2010.

[19] X. Lin and N. B. Shroff. Joint rate control and scheduling in multihop wireless networks. Proc. of 43rd IEEE Conf. on Decision and Control, Paradise Island, Bahamas, Dec. 2004.

[20] R. Agrawal and V. Subramanian. Optimality of certain channel aware scheduling policies. Proc. 40th Annual Allerton Conference on Communication, Control, and Computing, Monticello, IL, Oct. 2002.

[21] H. Kushner and P. Whiting. Asymptotic properties of proportionalfair sharing algorithms. Proc. of 40th Annual Allerton Conf. on Communication, Control, and Computing, 2002.

[22] C.-P. Li and M. J. Neely. Network utility maximization over partially observable markovian channels. Arxiv Technical Report: arXiv:1008.3421v1, Aug. 2010. 
[23] B. Liu, P. Terlecky, A. Bar-Noy, R. Govindan, and M. J. Neely. Optimizing information credibility in social swarming applications. ArXiv technical report, arXiv:1009:6006, Sept. 2010.

[24] D. Williams. Probability with Martingales. Cambridge Mathematical Textbooks, Cambridge University Press, 1991.

[25] M. J. Neely. Queue stability and probability 1 convergence via lyapunov optimization. Arxiv Technical Report, Oct. 2010.

[26] D. P. Bertsekas and J. N. Tsitsiklis. Neuro-Dynamic Programming. Athena Scientific, Belmont, Mass, 1996.

[27] M. J. Neely. Max weight learning algorithms with application to scheduling in unknown environments. arXiv:0902.0630v1, Feb. 2009.

[28] C. Bisdikian, L. M. Kaplan, M. B. Srivastava, D. J. Thornley, D. Verma, and R. I. Young. Building principles for a quality of information specification for sensor information. 12th Int'l Conf. on Information Fusion (Fusion '09), Seattle, WA, July 2009. 